\title{
A COMPREHENSIVE COMPARISON OF THE SUN TO OTHER STARS: SEARCHING FOR SELF-SELECTION EFFECTS
}

\author{
José A. Robles, ${ }^{1}$ Charles H. Lineweaver, ${ }^{1}$ Daniel Grether,${ }^{2}$ Chris Flynn, ${ }^{3}$ Chas A. Egan, ${ }^{2,4}$ \\ Michael B. Pracy, ${ }^{4}$ Johan Holmberg, ${ }^{5}$ and Esko Gardner ${ }^{3}$ \\ Received 2007 December 21; accepted 2008 May 10
}

\begin{abstract}
If the origin of life and the evolution of observers on a planet is favored by atypical properties of a planet's host star, we would expect our Sun to be atypical with respect to such properties. The Sun has been described by previous studies as both typical and atypical. In an effort to reduce this ambiguity and quantify how typical the Sun is, we identify 11 maximally independent properties that have plausible correlations with habitability and that have been observed by, or can be derived from, sufficiently large, currently available, and representative stellar surveys. By comparing solar values for the 11 properties to the resultant stellar distributions, we make the most comprehensive comparison of the Sun to other stars. The two most atypical properties of the Sun are its mass and orbit. The Sun is more massive than $95 \% \pm 2 \%$ of nearby stars, and its orbit around the Galaxy is less eccentric than $93 \% \pm 1 \%$ of FGK stars within $40 \mathrm{pc}$. Despite these apparently atypical properties, a $\chi^{2}$ analysis of the Sun's values for 11 properties, taken together, yields a solar $\chi_{\odot}^{2}=8.39 \pm 0.96$. If a star is chosen at random, the probability that it will have a lower value (i.e., be more typical) than the Sun, with respect to the 11 properties analyzed here, is only $29 \% \pm 11 \%$. These values quantify, and are consistent with, the idea that the Sun is a typical star. If we have sampled all reasonable properties associated with habitability, our result suggests that there are no special requirements for a star to host a planet with life.
\end{abstract}

Subject headings: stars: fundamental parameters — stars: statistics — Sun: fundamental parameters Sun: general

\section{INTRODUCTION}

If the properties of the Sun are consistent with the idea that the Sun was randomly selected from all stars, this would indicate that life needs nothing special from its host star and would support the idea that life may be common in the universe. More particularly, if there is nothing special about the Sun, we have little reason to limit our life-hunting efforts to planets orbiting Sunlike stars. As an example of the type of anthropic reasoning we are using, consider the following situation. Suppose uranium (a low-abundance element in the solar system and in the universe) was central to the biochemistry of life on Earth. Further, suppose that a comparison of our Sun to other stars showed that the Sun had more uranium than any other star. How should we interpret this fact? The most reasonable way to proceed would be to try to evaluate the probability that such a coincidence happened by chance and to determine whether we are justified in reading some importance into it. Although a correlation does not necessarily imply cause, we think that a correlation between the Sun's anomalous feature and life's fundamental chemistry would give us important clues about the conditions necessary for life. Specifically, the search for life around other stars as envisioned by the NASA's Terrestrial Planet Finder or ESA's Darwin Project and as currently underway with SETI would change the strategy to focus on the most uranium-rich stars. Another example: Suppose the Sun had the highest $[\mathrm{Fe} / \mathrm{H}]$ of all the stars that had ever been observed.

\footnotetext{
${ }^{1}$ Planetary Science Institute, Research School of Astronomy and Astrophysics, and Research School of Earth Sciences, The Australian National University, Canberra Australia; josan@mso.anu.edu.au.

${ }^{2}$ University of New South Wales, Sydney, Australia.

3 Tuorla Observatory, University of Turku, Finland.

${ }^{4}$ Research School of Astronomy and Astrophysics, The Australian National University, Canberra, Australia.

5 Max-Planck-Institut für Astronomie, Heidelberg, Germany.
}

Then high $[\mathrm{Fe} / \mathrm{H}]$ would be strongly implicated as a precondition for our existence, possibly by playing a crucial role in terrestrial planet formation. These are exaggerated examples of the more subtle correlations that a detailed and comprehensive comparison of the Sun with other stars could reveal.

Whether the Sun is a typical or atypical star with respect to one or a few properties has been addressed in previous studies. Using an approach similar to ours (comparing solar to stellar properties from particular samples), some studies have suggested that the Sun is a typical star (Gustafsson 1998; Allende Prieto 2006), while other studies have suggested that the Sun is an atypical star (Gonzalez 1999a, 1999b; Gonzalez et al. 2001). This apparent disagreement arises from three problems:

1. The language used to describe whether the Sun is or is not typical is often confusingly qualitative. For example, reporting the Sun as "metal-rich" can mean that the Sun is significantly more metal-rich than other stars (e.g., more metal-rich than $80 \%$ of other stars) or it can mean that the Sun is insignificantly metalrich (e.g., more metal-rich than $51 \%$ of other stars).

2. The stellar samples chosen for the comparison can be biased with respect to the property of interest.

3. The inclusion (or exclusion) of stellar properties for which it is suspected or known that the Sun is atypical will make the Sun appear more atypical (or typical).

In this paper we address problem 1 by using only quantitative measures when comparing the Sun's properties to other stars. Our main interest is to move beyond the qualitative assessment of the Sun as either typical or atypical and obtain a more precise quantification of the degree of the Sun's (a)typicality. In other words, we want to answer the question, "how typical is the Sun?" rather than "is the Sun typical or not?" There are at least two ways to quantify how typical the Sun is. This can be done for individual parameters by determining how many stars have values below or 
TABLE 1

Samples Used to Produce the Stellar Distributions Plotted in Figures 1-10

\begin{tabular}{|c|c|c|c|c|c|c|c|c|c|}
\hline Figure & Property & Range & Median $\mu_{1 / 2}$ & $\sigma_{68}{ }^{\mathrm{a}}$ & Solar Value & $\begin{array}{l}\text { Number } \\
\text { of Stars }\end{array}$ & Spectral type & $\begin{array}{l}d_{\max } \\
(\mathrm{pc})\end{array}$ & Source \\
\hline 1.................. & $\operatorname{Mass}\left(M_{\odot}\right)$ & $0.08-2$ & 0.33 & 0.37 & 1 & 125 & $\mathrm{~A} 1-\mathrm{M} 7$ & 7.1 & RECONS \\
\hline $3 \ldots \ldots \ldots \ldots \ldots \ldots$ & {$[\mathrm{Fe} / \mathrm{H}]$} & -1.20 to +0.46 & -0.08 & 0.20 & 0 & 453 & $\mathrm{~F} 7-\mathrm{K} 3$ & 25 & Grether \& Lineweaver (2007) \\
\hline 4A ..................... & {$[\mathrm{C} / \mathrm{O}]$} & -0.22 to +0.32 & 0.07 & 0.09 & 0 & 256 & FG & 150 & $\mathrm{G} 99, \mathrm{R} 03, \mathrm{BF} 06^{\mathrm{c}}$ \\
\hline 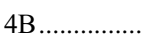 & {$[\mathrm{Mg} / \mathrm{Si}]$} & -0.18 to +0.14 & 0.01 & 0.04 & 0 & 231 & FG & 150 & $\mathrm{R} 03, \mathrm{~B} 05^{\mathrm{d}}$ \\
\hline 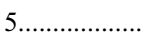 & $v \sin i\left(\mathrm{~km} \mathrm{~s}^{-1}\right)$ & $0-36$ & 2.51 & 1.27 & $1.28^{\mathrm{e}}$ & 276 & $\mathrm{~F} 8-\mathrm{K} 2$ & 80 & Valenti \& Fischer $(2005)^{\mathrm{f}}$ \\
\hline $8 \ldots \ldots \ldots \ldots \ldots \ldots$ & $R_{\mathrm{Gal}}(\mathrm{kpc})$ & $0-30$ & 4.9 & 5.03 & $7.62 \pm 0.32^{\mathrm{j}}$ & $\cdots$ & 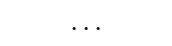 & 50,000 & $\mathrm{BS} 80, \mathrm{G} 96, \mathrm{E} 05^{\mathrm{k}}$ \\
\hline $9 \ldots \ldots \ldots \ldots \ldots \ldots$ & $M_{\text {gal }}\left(M_{\odot}\right)^{1}$ & $10^{7}-10^{12}$ & $10^{10.2}$ & 0.47 & $10^{10.55 \pm 0.16}$ & $\cdots$ & . & $10^{7}$ & D94, CB99, L00, BJ01, J03 \\
\hline $10 \ldots \ldots \ldots \ldots \ldots$ & $M_{\text {group }}\left(M_{\odot}\right)^{1}$ & $10^{9}-10^{13}$ & $10^{11.1}$ & 0.47 & $10^{10.91 \pm 0.07}$ & $\ldots$ & $\ldots$ & $10^{7}$ & Eke et al. (2004) \\
\hline
\end{tabular}

${ }^{a}$ Characteristic width of distribution in the direction of the solar value.

b Wright et al. (2004; see footnote 8 of this paper).

c G99: Gustafsson et al. (1999); R03: Reddy et al. (2003); BF06: Bensby \& Feltzing (2006).

${ }^{d}$ R03: Reddy et al. (2003); B05: Bensby et al. (2005).

e Solar rotational velocity corrected for random inclination (see $\S 2.5$ ).

${ }^{\mathrm{f}}$ Subset of stars within the mass range $0.9 M_{\odot} \leq M \leq 1.1 M_{\odot}$.

g Calculated using the solar galactic motion (Dehnen \& Binney 1998) and the Galactic potential (see $\S 2.6$ ).

${ }^{\mathrm{h}}$ Subset of volume complete A5-K2 stars within $40 \mathrm{pc}$.

${ }^{i}$ Integrated solar orbit in the Galactic potential of Flynn et al. (1996; see $\S 2.6$ ).

${ }^{\mathrm{j}}$ Eisenhauer et al. (2005).

${ }^{\mathrm{k}}$ BS80: Bahcall \& Soneira (1980); G96: Gould et al. (1996); E05: Eisenhauer et al. (2005).

${ }^{1}$ Stellar mass, not total baryonic mass or total mass.

${ }^{m}$ D94: Driver et al. (1994); CB99: Courteau \& van den Bergh (1999); L00: Loveday (2000); BJ01: Bell \& de Jong (2001); J03: Jarrett et al. (2003).

above the solar value (Table 3 ). This can also be done by a joint analysis of multiple parameters (Table 2). If there are several subtle factors that have some influence over habitability, a quantitative joint analysis of the Sun's properties may allow us to identify these factors without invoking largely speculative arguments linking specific properties to habitability.

With respect to problem 2, most previous analyses have compared the Sun to subsets of Sun-like stars selected to be Sun-like with respect to one or more parameters. In such analyses, the Sun will appear typical with respect to any parameter(s) correlated with one of the preselected Sun-like parameters. For example, elemental abundances $[\mathrm{X} / \mathrm{H}]$ are correlated with metallicity $^{6}[\mathrm{Fe} / \mathrm{H}]$. The sample of Edvardsson et al. (1993a) was selected to have a wide range of $[\mathrm{Fe} / \mathrm{H}]$. This produced a metallicity distribution unrepresentative of stars in general. Recognizing this, Edvardsson et al. (1993a) conditioned on solar metallicity, $[\mathrm{Fe} / \mathrm{H}] \approx 0$, and then compared solar abundances for 12 elements to the abundances in a group of nearby stars with solar iron abundance, solar age, and solar galactocentric radius. They found the Sun to be "a quite typical star for its metallicity, age, and galactic orbit." Similarly, Gustafsson (1998) after comparing various properties of the Sun to solar-type stars (stars of similar mass and age) concluded that the Sun seems very normal for its mass and age: "The Sun, to a remarkable degree, is solar type." The stellar samples we use for comparison with the Sun are, in our judgment, the least-biased samples currently available for such a comparison.

To address problem 3 , in $\S 2$ we compare the Sun to other stars using a large number (11) of maximally independent properties with plausible correlations with habitability. These properties can be observed or derived for a sufficiently large, representative stellar sample (Table 1). Any property of the Sun or its environment that must be special to allow habitability would show up in our

\footnotetext{
${ }^{6}$ Metallicity: $[\mathrm{Fe} / \mathrm{H}]$ is the fractional abundance of Fe relative to hydrogen, compared to the same ratio in the Sun: $[\mathrm{Fe} / \mathrm{H}] \equiv \log (\mathrm{Fe} / \mathrm{H})_{\star}-\log (\mathrm{Fe} / \mathrm{H})_{\odot}$.
}

analysis. However, in contrast to previous analyses that have looked for solar anomalies with respect to individual properties, we perform a joint analysis that enables us to quantify how typical the solar values are, taken as a group. In $\S 3$, the differences between the solar values and the stellar samples' medians are used to perform first a simple and then an improved version of a $\chi^{2}$ analysis to estimate whether the solar values are characteristic of a star selected at random from the stellar samples. The results of our joint analysis are presented in Figure 13. We find that the solar values, taken as a group, are consistent with the Sun being a random star. However, there are important caveats to this interpretation associated with the compromise between the number of properties analyzed and their plausibility of being correlated with habitability. In $\S \S 5$ and 6 we discuss these caveats and summarize. We discuss the levels of correlation between our 11 properties in Appendix A.

\section{STELLAR SAMPLES AND SOLAR VALUES}

We are looking for a signal associated with a prerequisite for, or a property that favors, the origin and evolution of life (see Gustafsson [1998] for a brief discussion of this idea). If we indiscriminately include many properties with little or no plausible correlation with habitability, we run the risk of diluting any potential signal. If we choose only a few properties based on previous knowledge that the Sun is anomalous with respect to those properties, we are making a useful quantification, but we are unable to address problem 3 . We choose a middle ground and try to identify as many properties as we can that have some plausible association with habitability. This strategy is most sensitive if a few unknown stellar properties (among the ones being tested) contribute to the habitability of a terrestrial planet in orbit around a star.

An optimal quantitative comparison of the Sun to other stars would require an unbiased, large representative stellar sample from which independent distributions for as many properties as 
desired could be compared. Such a distribution for each property of interest would allow a straightforward analysis and outcome: the Sun is within $n \%$ of stars around the centroid of the $\mathrm{N}$-dimensional distribution. However, observational and sample selection effects prevent the assembly of such an ideal stellar sample.

In this study, we compare the Sun to other stars with respect to the following 11 basic physical properties: (1) mass, (2) age, (3) metallicity [Fe/H], (4) carbon-to-oxygen ratio [C/O], (5) magnesium-to-silicon ratio $[\mathrm{Mg} / \mathrm{Si}]$, (6) rotational velocity $v \sin i,(7)$ eccentricity of the star's galactic orbit $e,(8)$ maximum height to which the star rises above the galactic plane $Z_{\max }$, (9) mean galactocentric radius $R_{\text {Gal }}$, (10) the mass of the star's host galaxy $M_{\text {gal }}$, and (11) the mass of the star's host group of galaxies $M_{\text {group }}$. These 11 properties span a wide range of stellar and galactic factors that may be associated with habitability. We briefly discuss how each parameter might have a plausible correlation with habitability. For each property we have tried to assemble a large, representative sample of stars whose selection criteria is minimally biased with respect to that property. For each property the percentage of stars with values lower and higher than the solar value are computed. For properties 9,10 , and 11 , the uncertainties in the percentages are determined from the uncertainties of the distributions. For the rest of the properties, nominal uncertainties $\Delta$ on the percentages were calculated assuming a binomial distribution (e.g., Meyer 1975): $\Delta=\left(n_{\text {low }} \times n_{\text {high }} / N_{\text {tot }}\right)^{1 / 2}$, where $n_{\text {low }}\left(n_{\text {high }}\right)$ is the fraction of stars with a lower (higher) value than the Sun and $N_{\text {tot }}$ is the total number of stars in the sample. The solar value is indicated with the Sun symbol $(\odot)$ in all figures.

We compare the Sun and its environment to other stars and their environments. The analysis of these larger environmental contexts provides information about properties that otherwise could not be directly measured. For example, suppose the metallicity of the Sun were normal with respect to stars in the solar neighborhood, but that these stars as a group had an anomalously high metallicity with respect to the average metallicity of stars in the universe. This fact would strongly suggest that habitability is associated with high metallicity, but our comparison with only local stars would not pick this up. In the absence of an $[\mathrm{Fe} / \mathrm{H}]$ distribution for all stars in the universe, we use galactic mass as a convenient proxy for any such property that correlates with galaxy mass.

\subsection{Mass}

Mass is probably the single most important characteristic of a star. For a main-sequence star, mass determines luminosity, effective temperature, main-sequence lifetime, and the dimensions, UV insolation, and temporal stability of the circumstellar habitable zone (Kasting et al. 1993).

Low-mass stars are intrinsically dim. Thus, a complete sample of stars can only be obtained out to a distance of $\sim 7 \mathrm{pc}(\approx 23 \mathrm{lt}-\mathrm{yr})$. Figure 1 compares the mass of the Sun to the stellar mass distribution of the 125 nearest main-sequence stars within $7.1 \mathrm{pc}$, as compiled by the RECONS consortium. ${ }^{7}$ Overplotted is the stellar initial mass function (IMF; see Kroupa 2002, eqs. [4] and [5] and Table 1) normalized to 125 stars more massive than the brown dwarf limit of $0.08 M_{\odot}$. Since the IMF appears to be fairly universal (Kroupa \& Weidner 2005), these nearby comparison stars are representative of a much larger sample of stars. There is good agreement between the histogram and the IMF; the Sun is more massive than $95 \% \pm 2 \%$ of the nearest stars and more massive

\footnotetext{
${ }^{7}$ See RECONS database at http://www.recons.org/.
}

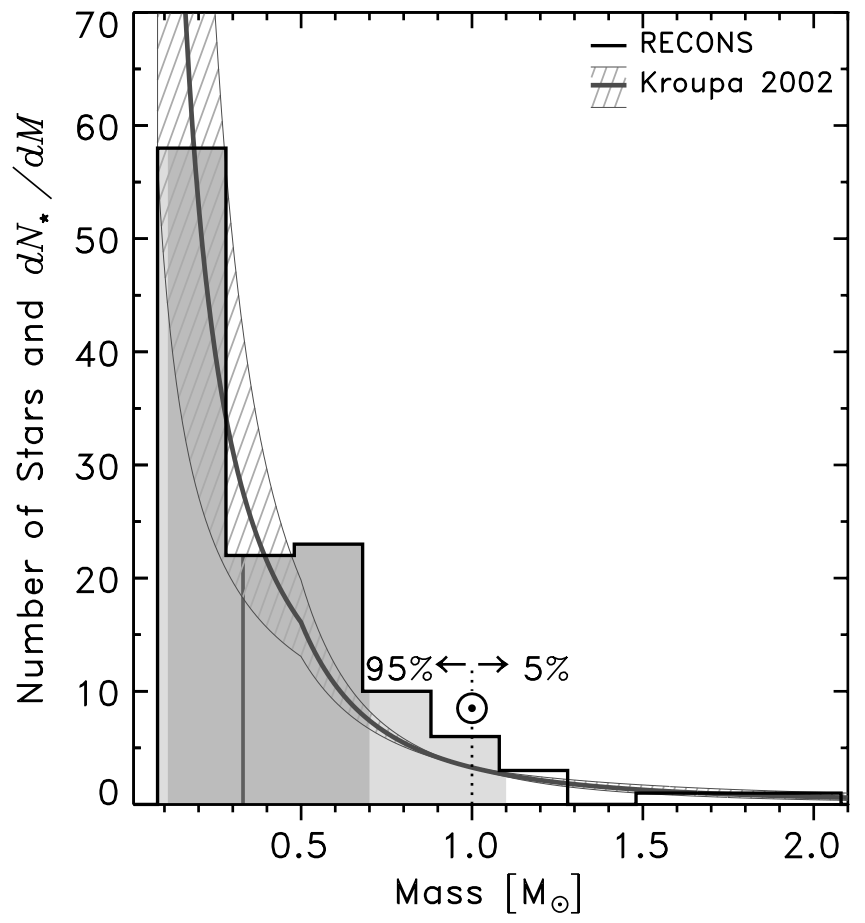

FIG. 1.-Mass histogram of the 125 nearest stars (see RECONS database, http://www.recons.org/). The median $\left(\mu_{1 / 2}=0.33 M_{\odot}\right)$ of the distribution is indicated by the vertical gray line. The $68 \%$ and $95 \%$ bands around the median are indicated, respectively, by the vertical dark gray and light gray bands. We also use these conventions in Figs. 2-11. The solid curve and hatched area around it represents the IMF and its associated uncertainty (Kroupa 2002). The Sun, indicated by the Sun symbol $(\odot)$, is more massive than $95 \% \pm 2 \%$ of these stars.

than $94 \% \pm 2 \%$ of the stars in the Kroupa (2002) IMF. Fourteen brown dwarfs and nine white dwarfs within $7.1 \mathrm{pc}$ were not included in this sample. Including them yields $94 \%$ - the same result obtained from the IMF. Our $95 \% \pm 2 \%$ result should be compared with the $91 \%$ reported by Gonzalez (1999b). The Sun's mass is the most anomalous of the properties studied here.

\subsection{Age}

If the evolution of observers like ourselves takes on average many billions of years, we might expect the Sun to be anomalously old (Carter 1983). Accurate estimation of stellar ages is difficult. For large stellar surveys (more than a few hundred stars), the most commonly used age indicators are based on isochrone fitting and/or chromospheric activity $\left(R_{\mathrm{HK}}^{\prime}\right.$ index $)$. Rocha-Pinto et al. (2000b) have estimated a star formation rate (SFR) or, equivalently, an age distribution for the local Galactic disk from chromospheric ages of 552 late-type (F8-K2) dwarf stars in the mass range $0.8 M_{\odot} \leq M \leq 1.4 M_{\odot}$ at distances $d \leq 200$ pc (RochaPinto et al. 2000a). They applied scale-height corrections, stellar evolution corrections, and volume incompleteness corrections that converted the observed age distribution into the total number of stars born at any given time. Hernandez et al. (2000) and Bertelli \& Nasi (2001) have made estimates of the star formation rate in the solar neighborhood and favor a smoother distribution (fewer bursts) than Rocha-Pinto et al. (2000b).

In Figure 2 we compare the chromospheric age of the $\operatorname{Sun}\left(\tau_{\odot}=\right.$ $4.9 \pm 3.0$ Gyr; Wright et al. 2004) $)^{8}$ to the stellar age distribution

${ }^{8}$ To ensure that the Sun's age is determined in the same way as the stellar ages to which it is being compared, we adopt the chromospheric solar age $\tau_{\odot}=4.9 \pm$ 3.0 Gyr over the more accurate meteoritic age $\tau_{\odot}=4.57 \pm 0.002$ Gyr (Allègre et al. 1995). 


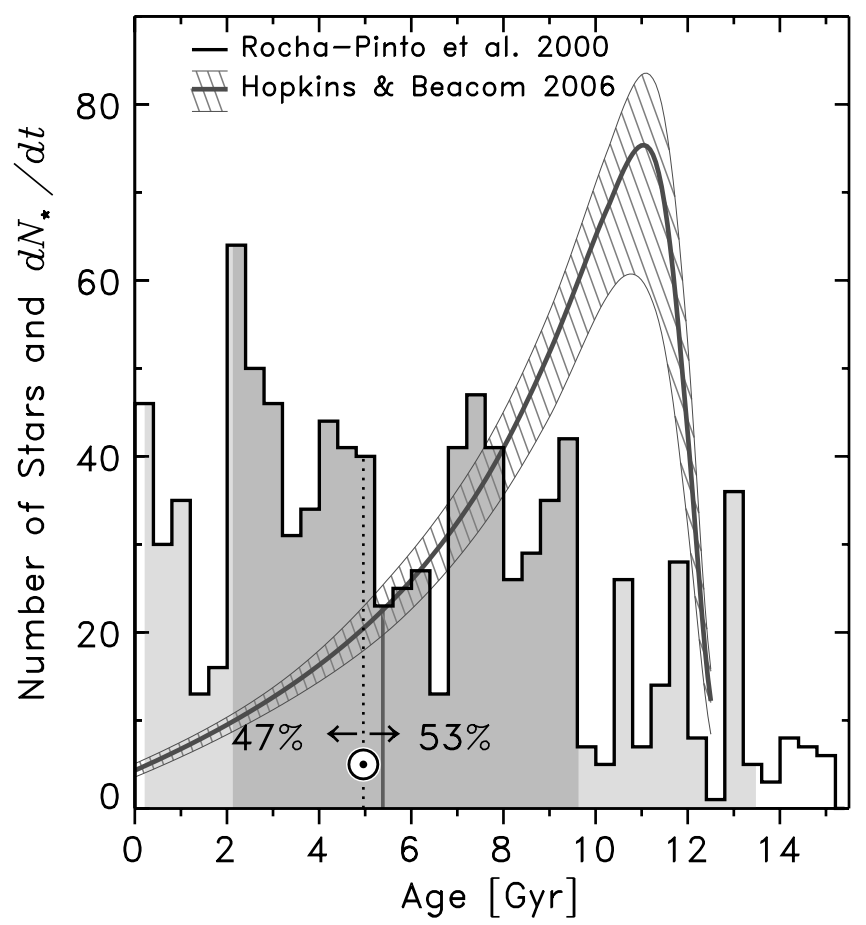

FIG. 2.-Galactic stellar age distribution (median $\mu_{1 / 2}=5.4$ Gyr) from RochaPinto et al. (2000b). The Sun is younger than $53 \% \pm 2 \%$ of the stars in the disk of our Galaxy. The gray curve is the cosmic SFR with its associated uncertainty (Hopkins \& Beacom 2006), according to which the Sun is younger than $86 \% \pm$ $5 \%$ of the stars in the universe.

representing the Galactic SFR (Rocha-Pinto et al. 2000b). The median of this distribution is 5.4 Gyr. The Sun is younger than $53 \% \pm 2 \%$ of the stars in the thin disk of our Galaxy. Overplotted is the cosmic SFR derived by Hopkins \& Beacom (2006). According to this distribution with a median $\mu_{1 / 2}=9.15 \mathrm{Gyr}$, the Sun was born after $86 \% \pm 5 \%$ of the stars that have ever been born.

The Galactic and cosmic SFRs are different because the cosmic SFR was dominated by bulges and elliptical galaxies in which the largest fraction of stellar mass in the universe resides. Bulges and elliptical galaxies (early-type galaxies) formed their stars early and quickly and then ran out of gas. The disks of spiral galaxies, like our Milky Way, seem to have undergone irregular bursts of star formation over a longer period of time as they interacted with their satellite galaxies.

The volume-limited ( $d_{\max }=40 \mathrm{pc}$ ) subset from Nordström et al. (2004) contains isochrone ages for 1126 A5-K2 stars. The median of this subset is $5.9 \mathrm{Gyr}$, and the Sun is younger than $55 \% \pm 2 \%$ of the stars. The similarity of this isochrone age result to the chromospheric age result is not obvious since the agreement between these two age techniques is rather poor. This mismatch can be seen in Figure 15D of Reid et al. (2007) and Figure 8 of Feltzing et al. (2001).

\subsection{Metallicity}

Iron is the most frequently measured element in nearby stars. Metallicity $[\mathrm{Fe} / \mathrm{H}]$ is known to be a proxy for the fraction of a star's mass that is not hydrogen or helium. In the Sun and possibly in the universe, the dominant contributors to this mass fraction in order of abundance are O (44\%), C (18\%), Fe (10\%), $\mathrm{Ne}(8 \%), \mathrm{Si}(6 \%), \mathrm{Mg}(5 \%), \mathrm{N}(5 \%)$, and S (3\%) (Asplund et al. 2005; Truran \& Heger 2005). The corresponding abundances by number are O (48\%), C (26\%), Ne (7\%), N (6\%), Mg (4\%), $\mathrm{Si}(4 \%), \mathrm{Fe}(3 \%)$, and $\mathrm{S}(2 \%)$. Importantly for this analysis, this short list contains the dominant elements in the composition of terrestrial planets $(\mathrm{O}, \mathrm{Fe}, \mathrm{Si}$, and $\mathrm{Mg})$ and life $(\mathrm{C}, \mathrm{O}, \mathrm{N}$, and $\mathrm{S})$.

Over the last few decades, much effort has gone into determining abundances in nearby stars for a wide range of elements. Stellar elemental abundances for element $\mathrm{X}$ are usually normalized to the solar abundance of the same element using a logarithmic abundance scale: $[\mathrm{X} / \mathrm{H}]_{\star} \equiv \log (\mathrm{X} / \mathrm{H})_{\star}-\log (\mathrm{X} / \mathrm{H})_{\odot}$. Hence, all solar elemental abundances $[\mathrm{X} / \mathrm{H}]_{\odot}$ are defined as zero. Spectroscopic abundance analyses are usually made differential relative to the Sun by analyzing the solar spectrum (reflected by the Moon, asteroids, or the telescope dome) in the same way as the spectrum of other stars. In this approach, biases introduced by the assumption of local thermodynamic equilibrium (LTE) largely cancel out for Sun-like stars (Edvardsson et al. 1993b).

A comparison between solar and stellar iron abundances is a common feature of most abundance surveys, and most have concluded that the Sun is metal-rich compared to other stars (Gustafsson 1998; Gonzalez 1999a, 1999b). However, for our purposes, the appropriateness of these comparisons depends on the selection criteria of the stellar sample to which the Sun has been compared. Stellar metallicity analyses such as Edvardsson et al. (1993a), Reddy et al. (2003), Nordström et al. (2004), and Valenti \& Fischer (2005) have stellar samples selected with different purposes in mind. For example, Edvardsson et al. (1993a) aimed to constrain the chemical evolution of the Galaxy, and their sample is biased toward low metallicity (average $[\mathrm{Fe} / \mathrm{H}]=-0.25$ ). The sample of Valenti \& Fischer 2005 (average $[\mathrm{Fe} / \mathrm{H}]=-0.01$ ) was selected as a planet candidate list and contains some bias toward high metallicity (see Grether \& Lineweaver 2007). To assess how typical the Sun is, Gustafsson (1998) limited the sample of Edvardsson et al. (1993a) to stars with galactocentric radii within $0.5 \mathrm{kpc}$ of the solar galactocentric radius, and to ages between 4 and 6 Gyr. The distribution of stars given by this criteria has an average $[\mathrm{Fe} / \mathrm{H}]=-0.09$.

Grether \& Lineweaver (2006, 2007) compiled a sample of 453 Sun-like stars within $25 \mathrm{pc}$. These stars were selected from the Hipparcos catalog, which is essentially complete to $25 \mathrm{pc}$ for stars within the spectral type range F7-K3 and absolute magnitude of $M_{V}=8.5$ (Reid 2002). Metallicities for this sample were assembled from a wide range of spectroscopic and photometric surveys. In Figure 3 we compare the Sun to the Grether \& Lineweaver (2007) sample, which has a median $[\mathrm{Fe} / \mathrm{H}]=-0.08$. To our knowledge this is the most complete and least-biased stellar spectroscopic metallicity distribution. The Sun is more metal-rich than $65 \% \pm 2 \%$ of these stars.

This result should be compared with that of Favata et al. (1997), who constructed a volume-limited $\left(d_{\max }=25 \mathrm{pc}\right)$ sample of $91 \mathrm{G}$ and $\mathrm{K}$ dwarfs ranging in color index $(B-V)$ between 0.5 and 0.8 (Favata et al. 1996). Their distribution has a median $[\mathrm{Fe} / \mathrm{H}]=$ -0.05 , and compared to this sample, the Sun is more metal-rich than $56 \% \pm 5 \%$ of the stars. Fuhrmann (2008) compared the Sun to a volume-complete $\left(d_{\max }=25 \mathrm{pc}\right)$ sample of about 185 thindisk mid-F-type to early K-type stars down to $M_{V}=6.0$. He finds a mean $[\mathrm{Fe} / \mathrm{H}]=-0.02 \pm 0.18$. This mean $[\mathrm{Fe} / \mathrm{H}]$ is lowered by 0.01 dex if the 43 double-lined spectroscopic binaries in his sample are included. His results are consistent with ours.

\subsection{Elemental Ratios $[\mathrm{C} / \mathrm{O}]$ and $[\mathrm{Mg} / \mathrm{Si}]$}

The elemental abundance ratios of a host star have a major impact on its protoplanetary disk chemistry and the chemical compositions of its planets. Oxygen and carbon make up $\sim 62 \%$ of the solar system's non-hydrogen-non-helium mass content $(Z=$ 0.0122; Asplund et al. 2005). Carbon and oxygen abundances are among the hardest to determine. This is due to high temperature 


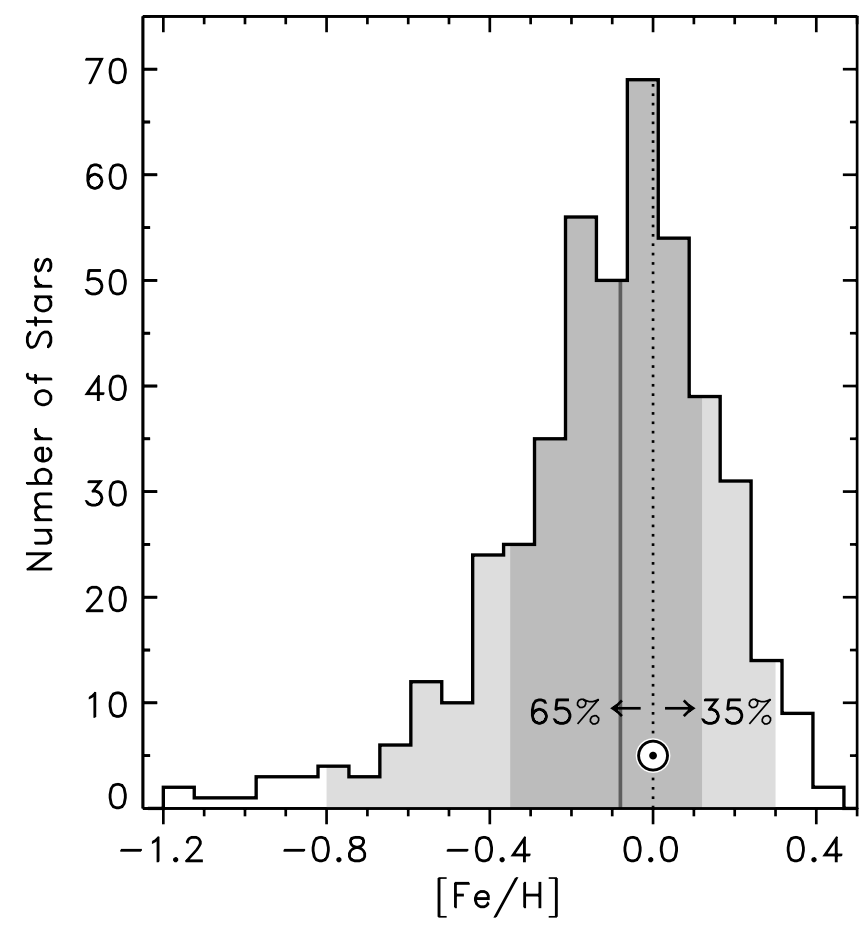

FIG. 3.- Stellar metallicity histogram of the 453 FGK Hipparcos stars within $25 \mathrm{pc}$ (Grether \& Lineweaver 2007). The median $\mu_{1 / 2}=-0.08$. The Sun is more metal-rich than $65 \% \pm 2 \%$ of the stars.

sensitivity and non-LTE effects in their permitted lines (e.g., $\mathrm{C}_{\mathrm{I}} 26588$, $\left.\mathrm{O}_{\mathrm{I}} 27773\right)$ and to the presence of blends in the for-

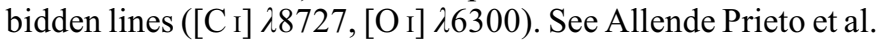
(2001) and Bensby \& Feltzing (2006) for details on $\mathrm{C}$ and $\mathrm{O}$ abundance derivations.

Carbon pairs up with oxygen to form carbon monoxide. In stars with a $\mathrm{C} / \mathrm{O}$ ratio larger than 1 , most of the oxygen condenses into $\mathrm{CO}$, which is largely driven out of the incipient circumstellar habitable zone by the stellar wind. In this oxygen-depleted scenario, planets formed within the snow line are formed in reducing environments and are mostly composed of carbon compounds, for example, silicon carbide (Kuchner \& Seager 2005). Thus, the $\mathrm{C} / \mathrm{O}$ ratio could be strongly associated with habitability.

As most heavy element abundances relative to hydrogen (e.g., $[\mathrm{O} / \mathrm{H}],[\mathrm{C} / \mathrm{H}],[\mathrm{N} / \mathrm{H}])$ are correlated with $[\mathrm{Fe} / \mathrm{H}]$, they were not included in our analysis. After the overall level of metallicity (represented by $[\mathrm{Fe} / \mathrm{H}]$ ), and after the ratio of the two most abundant metals, $[\mathrm{C} / \mathrm{O}]$, the magnesium to silicon ratio $[\mathrm{Mg} / \mathrm{Si}]$ is the most important ratio of the next most abundant elements (excluding the noble gas $\mathrm{Ne}$ ). For example, $[\mathrm{Mg} / \mathrm{Si}]$ sets the ratio of olivine to pyroxene, which determines the ability of a silicate mantle to retain water (H. O'Neill 2007, private communication).

Stellar elemental abundance ratios are defined as $\left[\mathrm{X}_{1} / \mathrm{X}_{2}\right]_{\star}=$ $\left[\mathrm{X}_{1} / \mathrm{H}\right]_{\star}-\left[\mathrm{X}_{2} / \mathrm{H}\right]_{\star}$. Hence, systematic errors associated with the determination of absolute solar abundances cancel for abundances relative to solar. We compile $[\mathrm{C} / \mathrm{O}]$ and $[\mathrm{Mg} / \mathrm{Si}]$ ratios from samples with the largest number of stars and highest signal-to-noise ratio stellar spectra:

1. [C/O]. - 256 stars from Gustafsson et al. (1999), Reddy et al. (2003), and Bensby \& Feltzing (2006).

2. $[\mathrm{Mg} / \mathrm{Si}]$. - 231 stars from Reddy et al. (2003) and Bensby et al. (2005).

Due to their selection criteria, these samples are biased toward low metallicity and therefore cannot be used to create a represen- tative $[\mathrm{Fe} / \mathrm{H}]$ distribution. Because a correlation exists between the $[\mathrm{C} / \mathrm{O}]$ and $[\mathrm{Mg} / \mathrm{Si}]$ ratios and $[\mathrm{Fe} / \mathrm{H}]$ (e.g., Gustafsson et al. 1999), the samples we use have a relatively narrow range of $[\mathrm{Fe} / \mathrm{H}]$ to reduce the influence of the correlation. Therefore, these small correlations can be neglected in this study; see the bottom panels of Figure 4, where $[\mathrm{Fe} / \mathrm{H}]$ versus $[\mathrm{C} / \mathrm{O}]$ as well as $[\mathrm{Fe} / \mathrm{H}]$ versus $[\mathrm{Mg} / \mathrm{Si}]$ are plotted. The top panels show the corresponding stellar distribution histograms. The Sun's [C/O] ratio is lower than $81 \% \pm 3 \%$ of the stars. This is consistent with Gonzalez (1999b), who suggested - based on data from Edvardsson et al. (1993a) and Gustafsson et al. (1999) — that the Sun has a low [C/O] ratio relative to Sun-like stars at similar galactocentric radii. See, however, Ramírez et al. (2007), who find that the Sun is oxygen-poor compared to solar metallicity stars.

The Sun's $[\mathrm{Mg} / \mathrm{Si}]$ ratio is lower than $66 \% \pm 3 \%$ of the stars. The $[\mathrm{C} / \mathrm{O}]$ and $[\mathrm{Mg} / \mathrm{Si}]$ ratios are also largely independent of each other (see Fig. 14 in Appendix A).

\subsection{Rotational Velocity}

Stellar rotational velocities are related to the specific angular momentum of a protoplanetary disk and possibly to the magnetic field strength of the star during planet formation, and to protoplanetary disk turbulence and mixing. An unusually low stellar rotational velocity may be associated with the presence of planets (Soderblom 1983). One or several of these factors could be related to habitability.

There is a known correlation between mass and $v \sin i$ at higher stellar masses (e.g., see Gray 2005, Fig. 18.21). In order to minimize the effect of this correlation (and maximize independence between parameters), we assembled a sample containing 276 stars within the mass range $0.9-1.1 M_{\odot}(\mathrm{F} 8-\mathrm{K} 2)$ from Valenti \& Fischer (2005). The selection criteria of the Valenti \& Fischer (2005) stars introduces some bias against more active stars. We compared the high $v \sin i$ tail of our Valenti \& Fischer (2005) sample with the high $v \sin i$ tail of a subsample from Nordström et al. (2004). We estimate that for our Valenti \& Fischer (2005) sample, the bias introduced by the selection criteria is lower than $\sim 5 \%$. The $v \sin i$ values in Valenti \& Fischer (2005) are obtained by fixing the macroturbulence for the stars of a given color without modeling the stars individually. If the macroturbulence value was underestimated for $T>5800 \mathrm{~K}$, the resulting $v \sin i$ values (especially when $v \sin i$ is near zero) would be overestimated (Valenti \& Fischer 2005, § 4).

The inclination of the stellar rotational axis to the line of sight is usually unknown, so the observable is $v \sin i$. Using the solar spectrum reflected by the asteroid Vesta, Valenti \& Fischer (2005) derived a solar $v \sin i=1.63 \mathrm{~km} \mathrm{~s}^{-1}$. For the purposes of this analysis we use the mean value that would be derived for the Sun when viewed from a random inclination: $v \sin i_{\odot}=1.63(\pi / 4) \mathrm{km} \mathrm{s}^{-1} \approx$ $1.28 \mathrm{~km} \mathrm{~s}^{-1}$.

The Sun rotates more slowly than $83 \% \pm 7 \%$ of the stars in our Valenti \& Fischer (2005) sample (Fig. 5). This is in agreement with Soderblom $(1983,1985)$, who reported that the Sun is within 1 standard deviation of stars of its mass and age.

\subsection{Galactic Orbital Parameters}

The Galactic velocity components of a $\operatorname{star}(U, V, W)$ with respect to the local standard of rest (LSR) may be used to compute a star's orbit in the Galaxy. How typical or atypical is the solar orbit compared to the orbits of other nearby stars in the Galaxy? The orbit may be related to habitability because more eccentric orbits bring a star closer to the Galactic center where there is a larger danger to life from supernova explosions, cosmic 

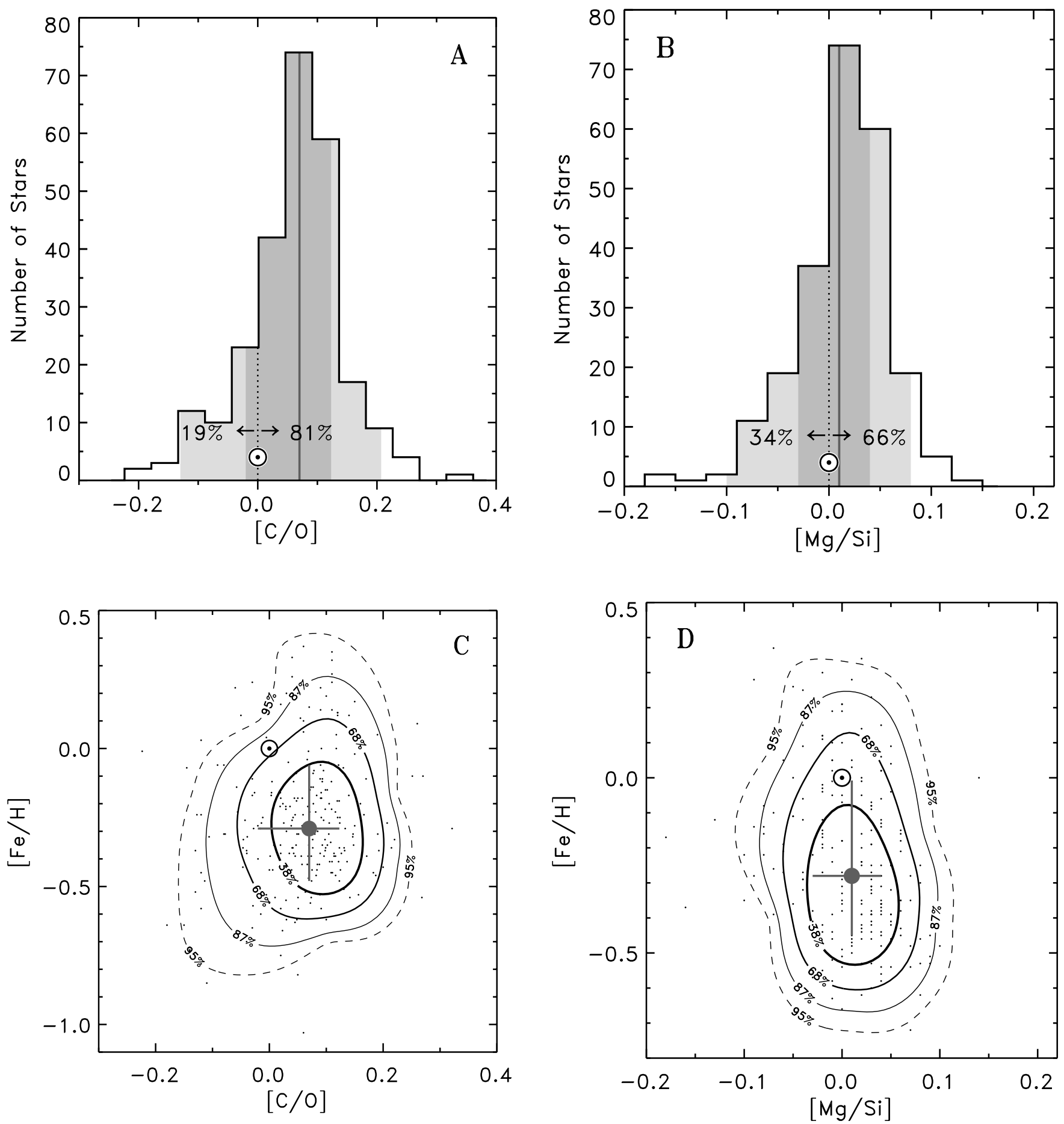

FIG. 4.-(A) Comparison of the Sun's carbon-to-oxygen ratio $\left([\mathrm{C} / \mathrm{O}]_{\odot} \equiv 0\right)$ to the $[\mathrm{C} / \mathrm{O}]$ ratios of 256 stars compiled from Gustafsson et al. (1999), Reddy et al. (2003), and Bensby \& Feltzing (2006). The Sun's [C/O] ratio is lower than $81 \% \pm 3 \%$ of the stars in this sample, which has a median $\mu_{12}=0.07$. (B) Comparison of the Sun's magnesium-to-silicon ratio $([\mathrm{Mg} / \mathrm{Si}] \odot \equiv 0)$, to $[\mathrm{Mg} / \mathrm{Si}]$ values from 231 stars from Reddy et al. (2003) and Bensby et al. (2005). The Sun's [ Mg/Si] ratio is lower than $66 \% \pm 3 \%$ of the stars in this sample with median $\mu_{1 / 2}=0.01$. (C, D) The small correlations of these distributions with [ $\left.\mathrm{Fe} / \mathrm{H}\right]$. These small correlations can be neglected for this study.

gamma and X-ray radiation, and any factors associated with higher stellar densities (Gonzalez et al. 2001; Lineweaver et al. 2004).

For a standard model of the Galactic potential, Nordström et al. (2004) computed orbital paramters for the Sun and for a large sample $(\sim 16,700)$ of A5-K2 stars. Their adopted components of the solar velocity relative to the local standard of rest were $(U, V, W)=(10.0 \pm 0.4,5.25 \pm 0.62,7.17 \pm 0.38) \mathrm{km} \mathrm{s}^{-1}$ (Dehnen \& Binney 1998).
For each of the 1987 stars within $40 \mathrm{pc}$ in the Nordström et al. (2004) catalog, inner and outer radii $R_{\min }$ and $R_{\max }$ were computed. This yielded the orbital eccentricity $e \equiv\left(R_{\max }-R_{\min }\right) /$ $\left(R_{\min }+R_{\max }\right)$. The solar eccentricity was computed using the components of the solar motion (Dehnen \& Binney 1998) relative to the local standard of rest in the Galactic potential of Flynn et al. (1996). The bottom panel of Figure 6 shows the correlation between Galactic orbital eccentricity $e$ and the magnitude of the galactic orbital velocities with respect to the local standard 


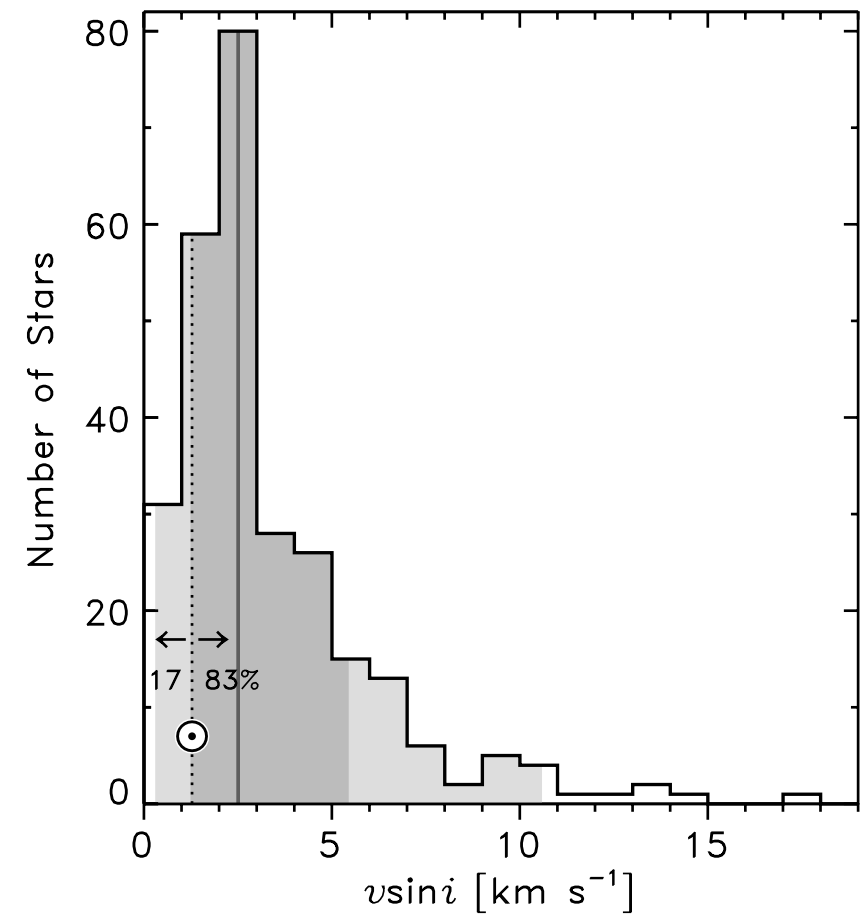

FIG. 5.- Rotational velocity histogram for 276 F8-K2 $\left(0.9 M_{\odot} \leq M \leq 1.1 M_{\odot}\right)$ stars (Valenti \& Fischer 2005). The Sun $\left(v \sin i_{\odot}=1.28 \mathrm{~km} \mathrm{~s}^{-1}\right)$ rotates more slowly than $83 \% \pm 7 \%$ of the stars. There is one star to the right of the plot with $v \sin i=36 \mathrm{~km} \mathrm{~s}^{-1}$.

of rest: $v_{\mathrm{LSR}} \equiv\left(U^{2}+V^{2}+W^{2}\right)^{1 / 2}$. Eccentricity $e$ and $v_{\mathrm{LSR}}$ are strongly correlated. We include $e$, not $v_{\text {LSR }}$, in the analysis since $e$ is less correlated with the maximum height above the Galactic plane $Z_{\max }$ than is $v_{\text {LSR }}$. This is shown in Figure 16 in Appendix A.

The Sun's eccentricity was determined with the same relation as the stellar eccentricities. The uncertainty in our estimate of solar eccentricity came from propagating the uncertainty in the adopted solar motion. We find $e_{\odot}=0.036 \pm 0.002$ (consistent with the $e_{\odot}=0.043 \pm 0.016$ found by Metzger et al. 1998). The Sun has a more circular orbit than $93 \% \pm 1 \%$ of the A5-K2 stars within $40 \mathrm{pc}$ (with median eccentricity $\mu_{1 / 2}=0.1$ ). This is the second most anomalous of the 11 solar properties we consider here.

The frequency of the passage of a star through the thin disk could be associated with Galactic gravitational tidal perturbations of Oort cloud objects that might increase the impact rate on potentially habitable planets. This is correlated with the maximum height, $Z_{\max }$, to which the stars rise above the Galactic plane. Figure 7 shows the stellar distribution of $Z_{\max }$ for the stars shown in Figure 6 . We find that $59 \% \pm 3 \%$ of the A5-K2 stars within $40 \mathrm{pc}$ of the Sun reach higher above the Galactic plane than the Sun does $\left(Z_{\max , \odot}=0.104 \pm 0.006 \mathrm{kpc}\right)$. The solar $Z_{\max , \odot}$ was derived by integrating the solar orbit in the Galactic potential. The uncertainty on $W$ produces the uncertainty on $Z_{\max , \odot}$ and hence the $\pm 3 \%$ uncertainty on $59 \%$. Our results for eccentricity and $Z_{\max }$ are consistent with those obtained using Hogg et al. (2005) LSR values: $(U, V, W)=(10.1 \pm 0.5,4.0 \pm 0.8,6.7 \pm$ 0.2 ). Using the Hogg et al. LSR values, $92 \% \pm 1 \%$ of A5-K2 stars within $40 \mathrm{pc}$ have higher eccentricities than the Sun and $62 \% \pm 4 \%$ of A5-K2 stars within 40 pc have larger $Z_{\max }$ values.

How does the Sun's distance from the center of the Milky Way compare to the distances of other stars from the center of the Milky Way? In Figure 8 we show the distribution of the mean radial distances of stars from the Galactic center, based on the star count model of Bahcall \& Soneira (1980). To represent the entire Galactic stellar population we include the disk (thin + thick) and spheroidal (bulge + halo) components. Using the current solar distance from the center $\left(R_{0}=7.62 \pm 0.32 \mathrm{kpc}\right.$; Eisenhauer et al. 2005) and a disk scale length $h=3.0 \pm 0.4 \mathrm{kpc}$ (Gould et al. 1996), we estimate that the Sun lies farther from the Galactic center than $72_{-5}^{+8} \%$ of the stars in the Galaxy. The uncertainty on
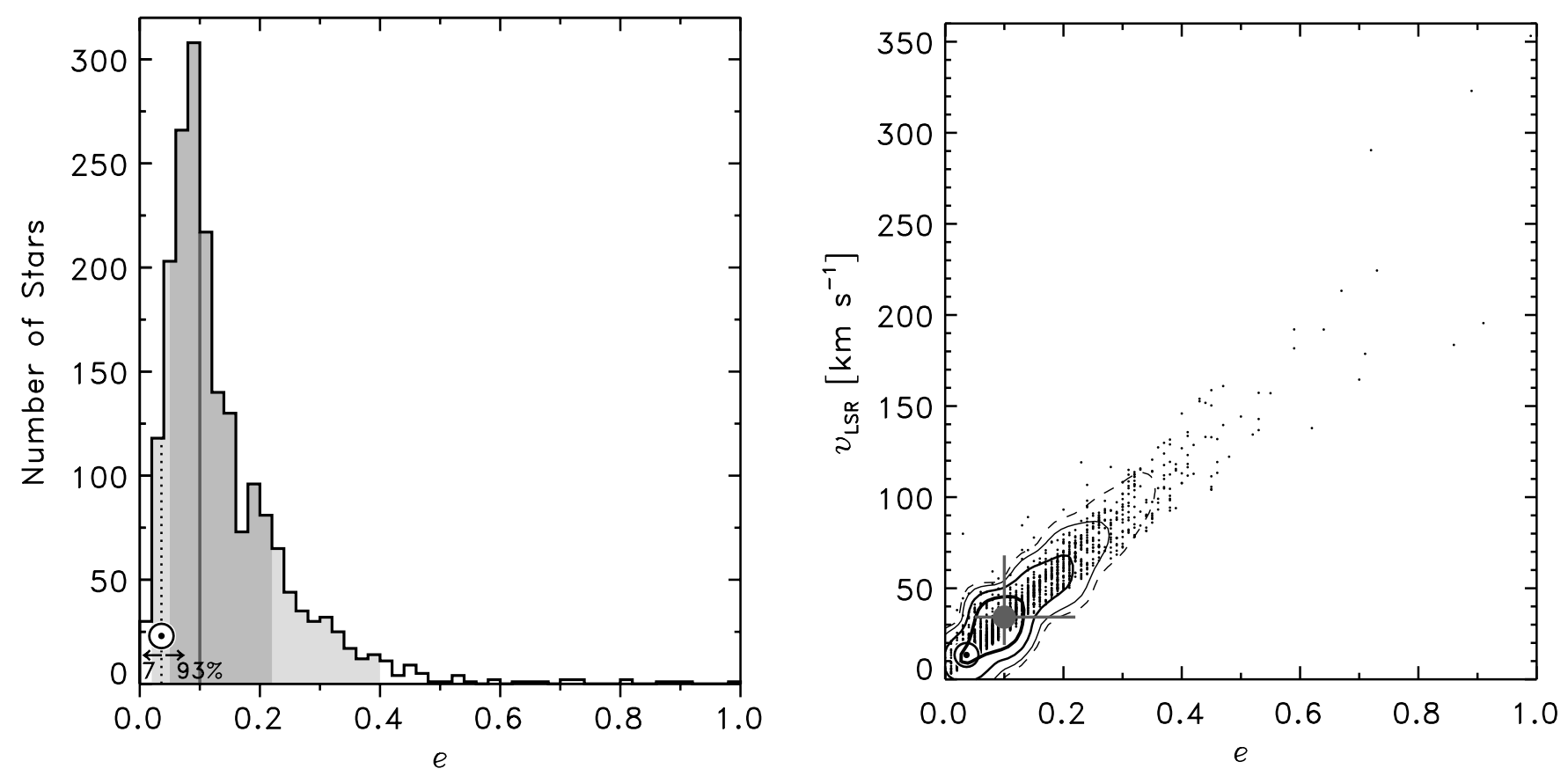

FIG. 6.-Top: Eccentricity distribution for the 1987 stars at $d \leq 40$ pc from Nordström et al. (2004). The Sun has a more circular orbit than $93 \% \pm 1 \%$ of the A5-K2

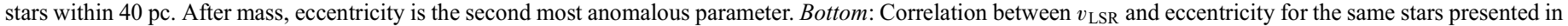

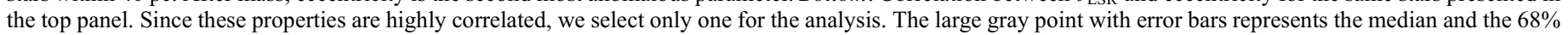
widths of the two one-dimensional distributions. As in Fig. 4, the contours correspond to $38 \%, 68 \%, 82 \%$, and $95 \%$. 


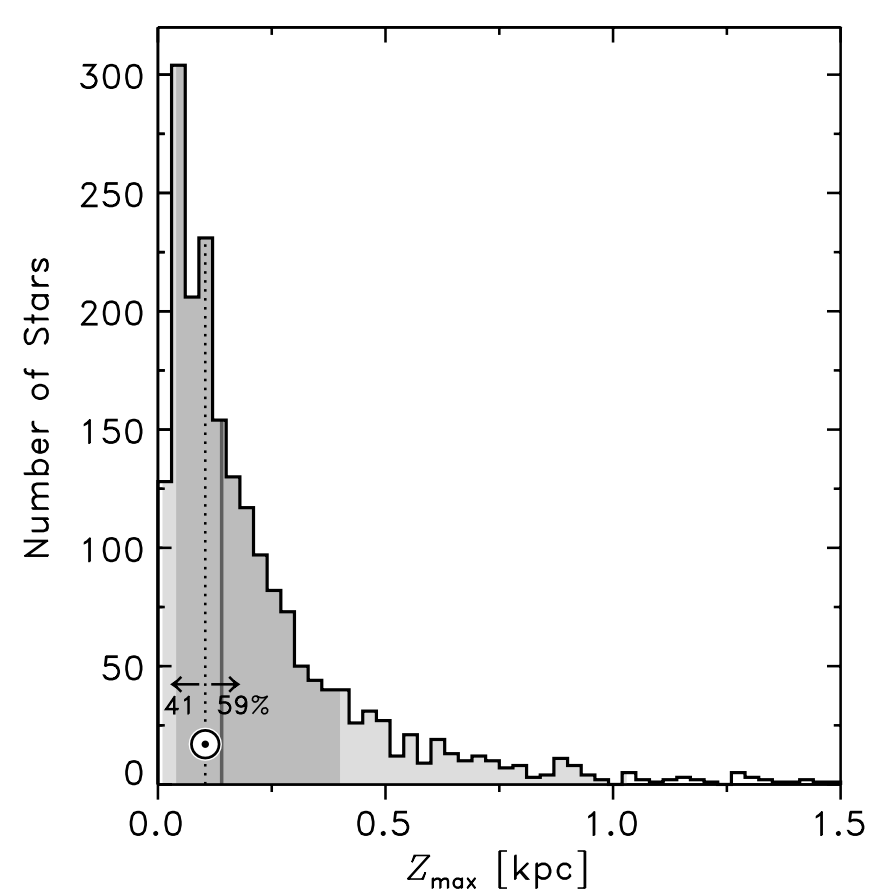

FIG. 7.-Distribution of maximum heights above the Galactic plane for the Nordström et al. (2004) sample. $59 \% \pm 3 \%$ of nearby A5-K2 stars $\left(d_{\max }=\right.$ $40 \mathrm{pc}$ ) reach higher above the Galactic plane than the Sun reaches. There are 22 stars evenly distributed over $Z_{\max }$ between 1.5 and $9.6 \mathrm{kpc}$. Their exclusion from the comparison reduces the $59 \%$ result by less than $1 \%$.

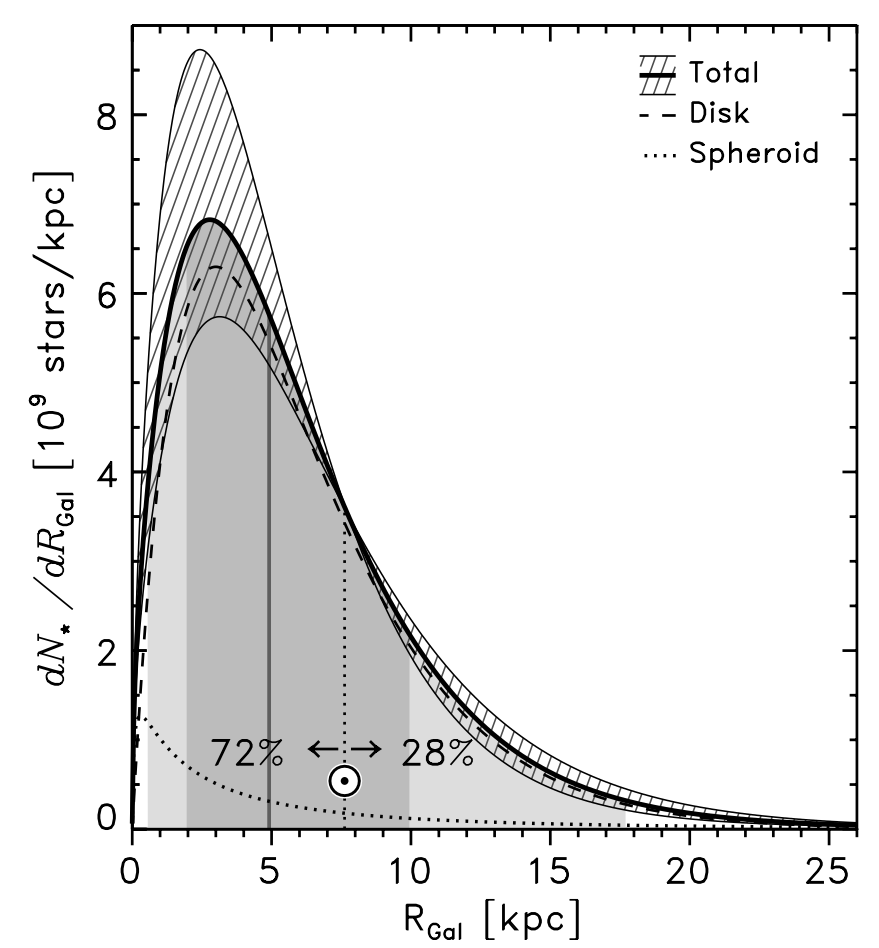

FIG. 8.- Mean stellar galactocentric radius distribution $d N_{\star} / d R_{\mathrm{Gal}}$. The solid curve represents the sum of the disk (dashed curve) and spheroidal (dotted curve) stellar components. The $68 \%$ uncertainty of the total distribution is shown by the cross-hatched area. The Sun is farther from the Galactic center than $72_{-5}^{+8} \%$ of the stars in the Galaxy.

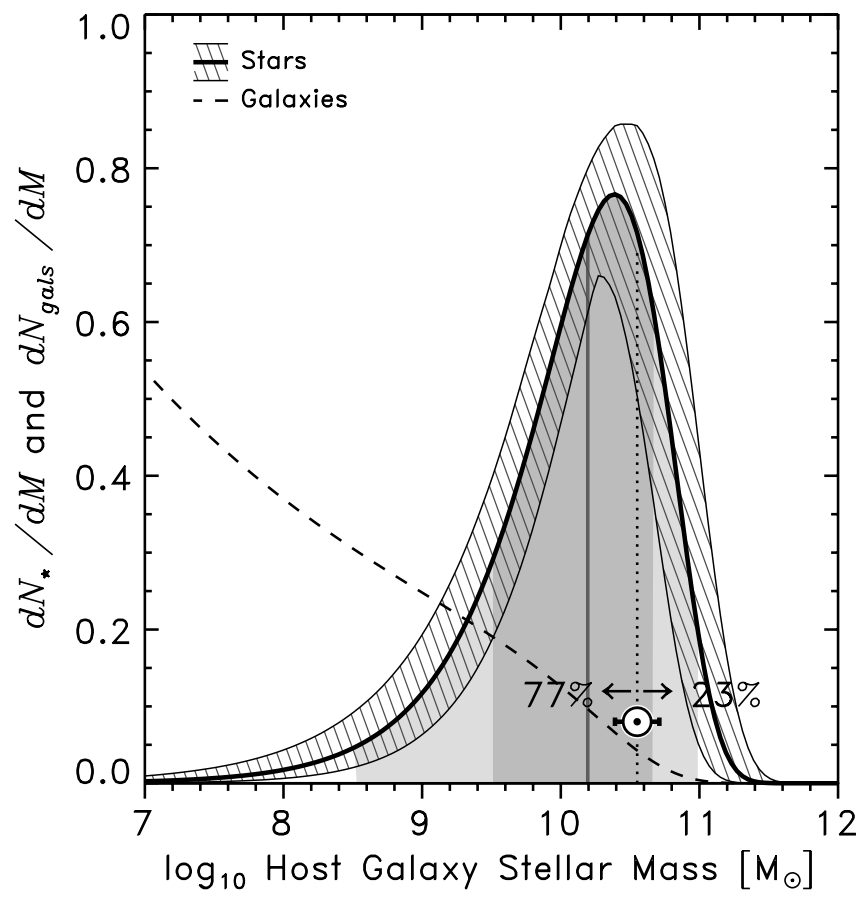

FIG. 9.- Fraction of all stars that live in galaxies of a given mass, $d N_{\star} / d M$ (solid curve). The mass of the Sun's galaxy is indicated by the Sun symbol $(\odot)$. This distribution represents the amount of stellar mass contributed by galaxies of a given mass. Approximately $77_{-14}^{+11 \%}$ of stars live in galaxies less massive than ours. The cross-hatched band shows the $1 \sigma$ uncertainty associated with the uncertainty in the two Schechter function parameters, $\alpha$ and $L^{*}$ (Loveday 2000; Schechter 1976). The dashed line shows the unweighted luminosity function (the number of galaxies per luminosity interval $d N_{\text {gals }} / d M$ ) according to which the Milky Way is more massive than $\sim 99 \%$ of galaxies.

the result comes from the $68 \%$ bounds of the total distribution, which come from the scale length uncertainty $( \pm 0.4 \mathrm{kpc})$.

\subsection{Host Galaxy Mass}

The mass of a star's host galaxy may be correlated with parameters that have an influence on habitability. For example, galaxy mass affects the overall metallicity distribution that a star would find around itself - an effect that would not show up in Figure 3, which only shows the local metallicity distribution.

The Milky Way is more massive than $\sim 99 \%$ of all galaxies; the precise fraction depends on the lower mass limit chosen for an object to be classified as a galaxy and the behavior of the lowmass end of the galaxy mass function (Silk 2007). We are referring here to the stellar mass, not the total baryonic mass or the total mass. Despite the Milky Way's large mass compared to other galaxies, if most stars in the universe resided in even more massive galaxies, the Milky Way would be a rather low mass galaxy for a star to belong to. To estimate the fraction of all stars in galaxies of a given mass, we first estimate the distribution of galaxy masses by taking the $K$-band luminosity function of Loveday (2000; the $K$ band most closely reflects stellar mass since it is less sensitive than other bands to differences in stellar populations) and weighting it by luminosity. We convert this to stellar mass assuming a constant stellar mass-to-light ratio of 0.5 (Bell $\&$ de Jong 2001). This function, plotted in Figure 9, shows the amount of stellar mass contributed by galaxies of a given massor assuming identical stellar populations - the fraction of stars residing in galaxies of a given stellar mass.

We estimate the $K$-band luminosity of the Milky Way by converting the published $V$-band magnitude of Courteau $\&$ van den Bergh (1999) to the $K$ band assuming the mean color of an Sbc 


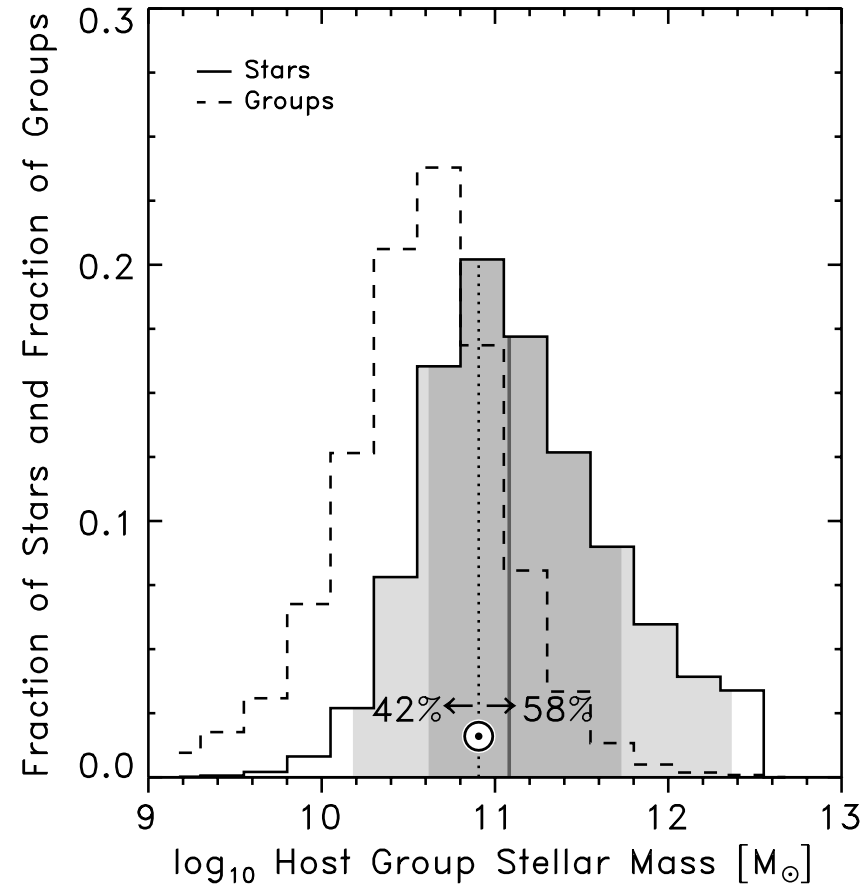

FIG. 10.-Dashed histogram shows the luminosity function of galactic groups (number of groups per interval of $B$-band luminosity). The solid histogram shows the luminosity-weighted group luminosity function (approximately the fraction of stars that inhabit a group of given stellar mass). The horizontal axis has been converted to stellar mass assuming a constant $B$-band stellar mass-to-light ratio of 1.5 (Bell \& de Jong 2001). The Sun symbol $(\odot)$ shows the estimated mass of the Local Group (Courteau \& van den Bergh 1999) and lies just below the median (vertical gray line).

spiral galaxy from the 2 MASS Large Galaxy Atlas (Jarrett et al. 2003) and applying the color conversion from Driver et al. (1994). We then convert this to stellar mass using the same stellar mass-tolight ratio used above, i.e., 0.5. In this way we estimate the stellar mass content of the Milky Way to be $10^{10.55 \pm 0.16}=3.6_{-1.1}^{+1.5} \times$ $10^{10} M_{\odot}$ (see also Flynn et al. 2006). Comparing this to the stellar masses of other galaxies (Fig. 9), we find that $77_{-14}^{+11 \%}$ of stars reside in galaxies less massive than the Milky Way.

\subsection{Host Group Mass}

The mass of a star's host galactic group or galactic cluster may be correlated with parameters that have an influence on habitability. For example, group mass is correlated with the density of the galactic environment (number of galaxies per $\mathrm{Mpc}^{3}$ ) that could, like galactocentric radius, be associated with the dangers of high stellar densities: "The presence of a giant elliptical at a distance of $50 \mathrm{kpc}$ would have disrupted the Milky Way Galaxy, so that human beings (and hence astronomers) probably would not have come into existence" (van den Bergh 2000). Our Local Group of galaxies seems rather typical (van den Bergh 2000), but we would like to quantify this. Proceeding similarly to our analysis of galaxy mass in $\S 2.7$, we ask, what fraction of stars live in galactic groups less massive than our Local Group? Figure 10 shows the luminosity-weighted (i.e., stellar mass-weighted) number density of galactic groups. The number distribution and luminosity distribution of galactic groups is taken from the Two-degree Field Galaxy Redshift Survey Percolation-Inferred Galaxy Group (2PIGG) catalog (Eke et al. 2004). It spans the range from weak groups to rich galaxy clusters.

We estimated the stellar masses of the 2PIGG groups and Local Group galaxies (Courteau \& van den Bergh 1999) by con- verting from the $B$ band assuming a constant stellar mass-to-light ratio of 1.5 (Bell \& de Jong 2001). This gives an estimated stellar mass of the local group of $10^{10.91 \pm 0.07}=8.1_{-1.2}^{+1.4} \times 10^{10} M_{\odot}$. Figure 10 indicates that our Local Group is a typical galactic grouping for a star to be part of. Approximately 58\% $\pm 5 \%$ of stars live in galactic groups more massive than our Local Group. With respect to the mass of its galaxy and the mass of its galactic group, the Sun is a fairly typical star in the universe.

\section{JOINT ANALYSIS OF 11 SOLAR PROPERTIES

$$
\text { 3.1. Solar } \chi^{2} \text { Analysis }
$$

We would like to know whether the solar properties, taken as a group, are consistent with noise, i.e., are they consistent with the values of a star selected at random from our stellar distributions. We take a $\chi^{2}$ approach to answering this question. First we estimate the solar $\chi_{\odot}^{2}$ by adding in quadrature, for all 11 properties, the differences between the solar values and the median stellar values. We find

$$
\chi_{\odot}^{2}=\sum_{i=1}^{N=11} \frac{\left(x_{\odot, i}-\mu_{1 / 2, i}\right)^{2}}{\sigma_{68, i}^{2}}=7.88_{-0.30}^{+0.08},
$$

where $i$ is the property index, $N=11$ is the number of properties we are considering, $\mu_{1 / 2, i}$ is the median of the $i^{t h}$ stellar distribution, and $\sigma_{68, i}$ is the difference between the median and the upper or lower $68 \%$ zone, depending on whether the solar value $x_{\odot, i}$ is above or below the median. The uncertainty on $\chi_{\odot}^{2}$ is obtained using the uncertainties of $x_{\odot, i}$.

Equation (1) can be improved on by taking into account (1) the non-Gaussian shapes of the stellar distributions and (2) the larger uncertainties of the medians of smaller samples (our smallest sample is $\sim 100$ stars).

We employ a bootstrap analysis (Efron 1979) to randomly resample data (with replacement) and derive a more accurate estimate of $\chi_{\odot}^{2}$. Because the bootstrap is a nonparametric method, the distributions need not be Gaussian.

We obtain $\chi_{\odot}^{2}=8.39 \pm 0.96$. Figure 11 shows the resulting solar $\chi^{2}$ distribution. The median of this distribution is our adopted solar $\chi^{2}$ value. Dividing our adopted solar $\chi^{2}$ by the number of degrees of freedom gives our adopted reduced solar $\chi^{2}$ value:

$$
\chi_{\odot}^{2} / 11=0.76 \pm 0.09 .
$$

The standard conversion of this into a probability of finding a lower $\chi^{2}$ value (assuming normally distributed independent variables) yields

$$
\begin{gathered}
P\left(<\chi_{\odot}^{2}=8.39 \mid N=11\right)=0.32 \pm 0.09 . \\
\text { 3.2. Estimate of } P\left(<\chi_{\odot}^{2}\right)
\end{gathered}
$$

To quantify how typical the Sun is with respect to our 11 properties, we compare the solar $\chi_{\odot}^{2}(=8.39)$ to the distribution of $\chi^{2}$ values obtained from the other stars in the samples.

We perform a Monte Carlo simulation (Metropolis \& Ulam $1949)$ to calculate an estimate of each star's $\chi^{2}$ value $\left(\chi_{\star}^{2}\right)$. The histogram shown in Figure 12 is the resulting Monte Carlo stellar $\chi^{2}$ distribution. Three standard $\chi^{2}$ distributions have been overplotted for comparison $(N=10,11,12)$. The probability of finding a star with $\chi^{2}$ lower than or equal to solar is

$$
P_{\mathrm{MC}}\left(\leq \chi_{\odot}^{2}=8.39 \mid N=11\right)=0.29 \pm 0.11 \text {. }
$$

The Monte Carlo $\chi^{2}$ distribution has a similar shape to the standard $\chi^{2}$ distribution function for $N=11$, and thus both yield 


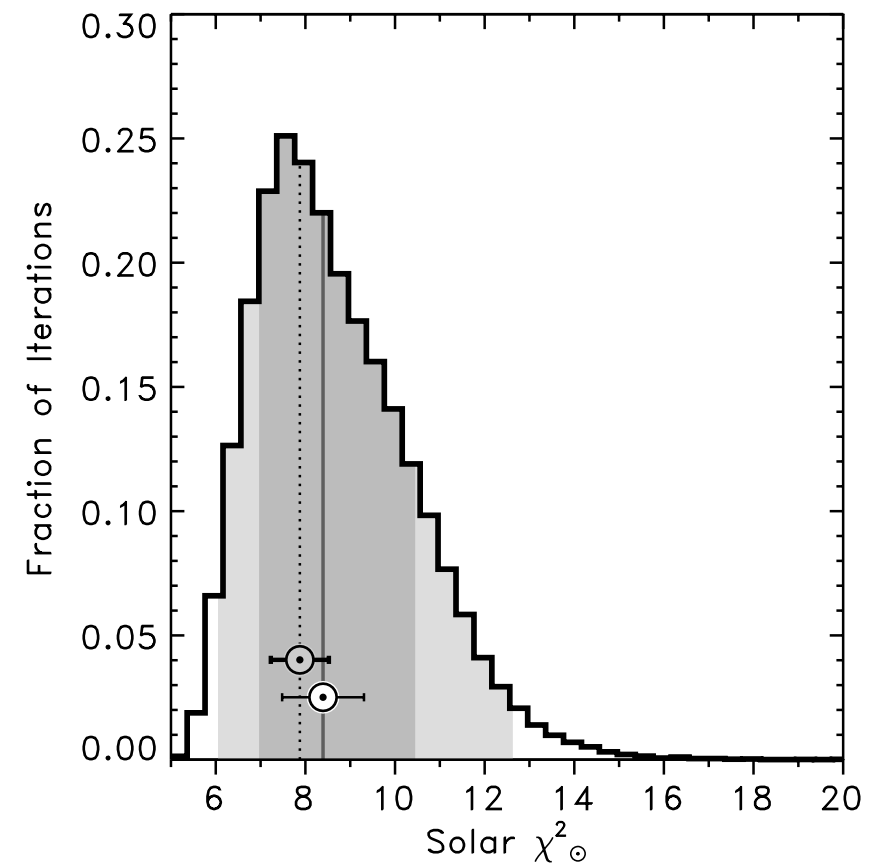

FIG. 11.-Bootstrapped solar $\chi^{2}$ distribution. The median of the distribution (white Sun symbol $[\odot]$ ) is $\chi_{\odot}^{2}=8.39 \pm 0.96$. This should be compared to the

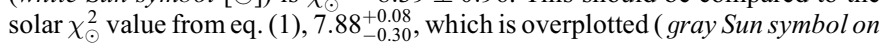
dotted line).

similar probabilities: $P_{\mathrm{MC}}\left(\leq \chi^{2}\right)=0.29 \sim P\left(\leq \chi^{2}\right)=0.32$ (eqs. [3] and [4]). The more appropriate Monte Carlo distribution has a longer tail, produced by the longer super-Gaussian tails of the stellar distributions.

Table 2 summarizes our analysis for the solar $\chi_{\odot}^{2}$ values and the probabilities $P\left(<\chi_{\odot}^{2}\right)$. Our simple $\chi_{\odot}^{2}=7.88$ estimate increased to 8.39 , and the uncertainty increased by a factor of $\sim 3$ after non-Gaussian and sample size effects were included as additional sources of uncertainty. Our improved analysis yields $P_{\mathrm{MC}}\left(\leq \chi_{\odot}^{2}\right)$, with a longer tail and brings the probability down from $0.32 \pm 0.09$ to $0.29 \pm 0.11$. If this value were close to 1 , almost all other stars would have lower $\chi^{2}$ values, and we would have good reason to suspect that the Sun is not a typical star. However, this preliminary low value of 0.29 indicates that if a star is chosen at random, the probability that it will be more typical (i.e., have a lower $\chi^{2}$ value) than the Sun (with respect to the 11 properties analyzed here) is only $29 \% \pm 11 \%$. The details of our improved estimates of $\chi_{\odot}^{2}$ and $P\left(<\chi_{\odot}^{2}\right)$ can be found in Appendix B.

\section{RESULTS}

Figure 13 shows four different representations of our results. Figure $13 a$ compares the solar values to each stellar distribution's median and $68 \%$ and $95 \%$ zones. The Sun lies beyond the $68 \%$ zone for three properties: mass (95\%), eccentricity (93\%), and rotational velocity $(88 \%)$. No solar property lies beyond the $95 \%$ zone. The histogram in Figure $13 b$ is the distribution of solar values in units of standard deviations:

$$
z_{i}=\frac{x_{\odot, i}-\mu_{1 / 2, i}}{\sigma_{68, i}}
$$

For each stellar property $i$, the Sun has a larger value than $n_{i} \%$ of the stars. If the Sun were a randomly selected star, we would

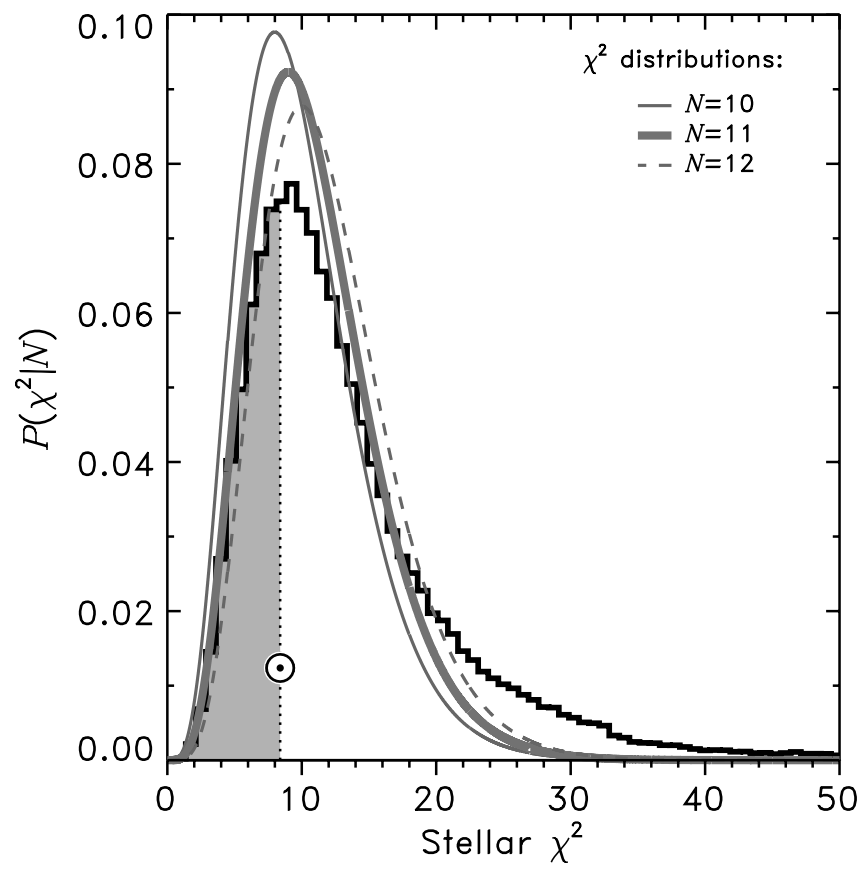

FIG. 12.- Stellar $\chi^{2}$ distribution from our Monte Carlo simulation. $P_{\mathrm{MC}}\left(<\chi_{\odot}^{2}=8.39\right)=0.29 \pm 0.11$ (gray scale) is calculated integrating from $\chi^{2}=0$ to $\chi^{2}=\chi^{2}$. For comparison, three $\chi^{2}$ distribution curves are overplotted with 10,11, and 12 degrees of freedom. The standard probability from the $N=11$ curve yields $P\left(<\chi_{\odot}^{2}=8.39 \mid N=11\right)=0.32 \pm 0.09$.

expect the percentages $n_{i} \%$ to be scattered roughly evenly between $0 \%$ and $100 \%$. When the $n_{i} \%$ values are lined up in decreasing order (Fig. 13c), we expect them to be near the line given by

$$
n_{i, \text { expected }} \%=\left[1-\frac{(i-1 / 2)}{N}\right] \times 100 \%
$$

and plotted in Figure 13c. Any anomalies would show up as Sun symbols significantly distant from the line.

Figure $13 d$ compares the percentages $n_{i} \%$ of stars having subsolar values (shown in Fig. 13c) with the solar values expressed in units of standard deviations from each distribution's median (shown in Fig. 13b). If the stellar distributions were perfect Gaussians, the translation from $z_{i}$ to $n_{i}$ would be given by the cumulative Gaussian distribution (Fig. 13d, solid curve). That the points lie along this line demonstrates that the approximation of our distributions as Gaussians is reasonable.

Table 3 lists percentages $n_{i} \%$ of stars for each property (as shown in Fig. 13). In the lower half of the table we list properties not included in this analysis because of correlations with properties that are included.

Individual stellar uncertainties make the observed characteristic widths $\left(\sigma_{68}\right.$, Table 1, col. [5]) larger than the widths of the intrinsic distributions. This broadening effect makes the Sun appear more typical than it really is when $\sigma_{68}$ and the individual stellar uncertainties $\left(\sigma_{\star}\right)$ are of similar size and the individual stellar uncertainties are much larger than the solar uncertainty $\left(\sigma_{\odot}\right)$. We estimate that our results are not significantly affected by this broadening effect.

Our resulting probability of finding a star with a $\chi^{2}$ lower or equal to the solar value of $29 \% \pm 11 \%$ (eq. [4]) is consistent with the probability we would obtain if stellar multiplicity were included in our study. Using the volume-limited sample used for stellar mass in $\S 2.1$ (125 A1-M7 stars within $7.1 \mathrm{pc})$ the 
TABLE 2

Summary of $\chi^{2}$ and $P\left(<\chi_{\odot}^{2}\right)$ Results

\begin{tabular}{ccccc}
\hline \hline Analysis & $\chi_{\odot}^{2}$ & $\chi_{\odot}^{2} / 11$ & $P\left(<\chi_{\odot}^{2} \mid N=11\right)$ & $P_{\mathrm{MC}}\left(<\chi_{\odot}^{2} \mid N=11\right)$ \\
\hline Simple ........................ & $7.88_{-0.30}^{+0.08}$ (eq. [1]) & $0.72_{-0.03}^{+0.01}$ & $0.28_{-0.03}^{+0.01}$ (Eq. [B1]) & $\ldots$ \\
Improved ..................... & $8.39 \pm 0.96$ & $0.76 \pm 0.09$ (eq. [2]) & $0.32 \pm 0.09$ (eq. [3]) & $0.29 \pm 0.11$ (eq. [4]) \\
\hline
\end{tabular}
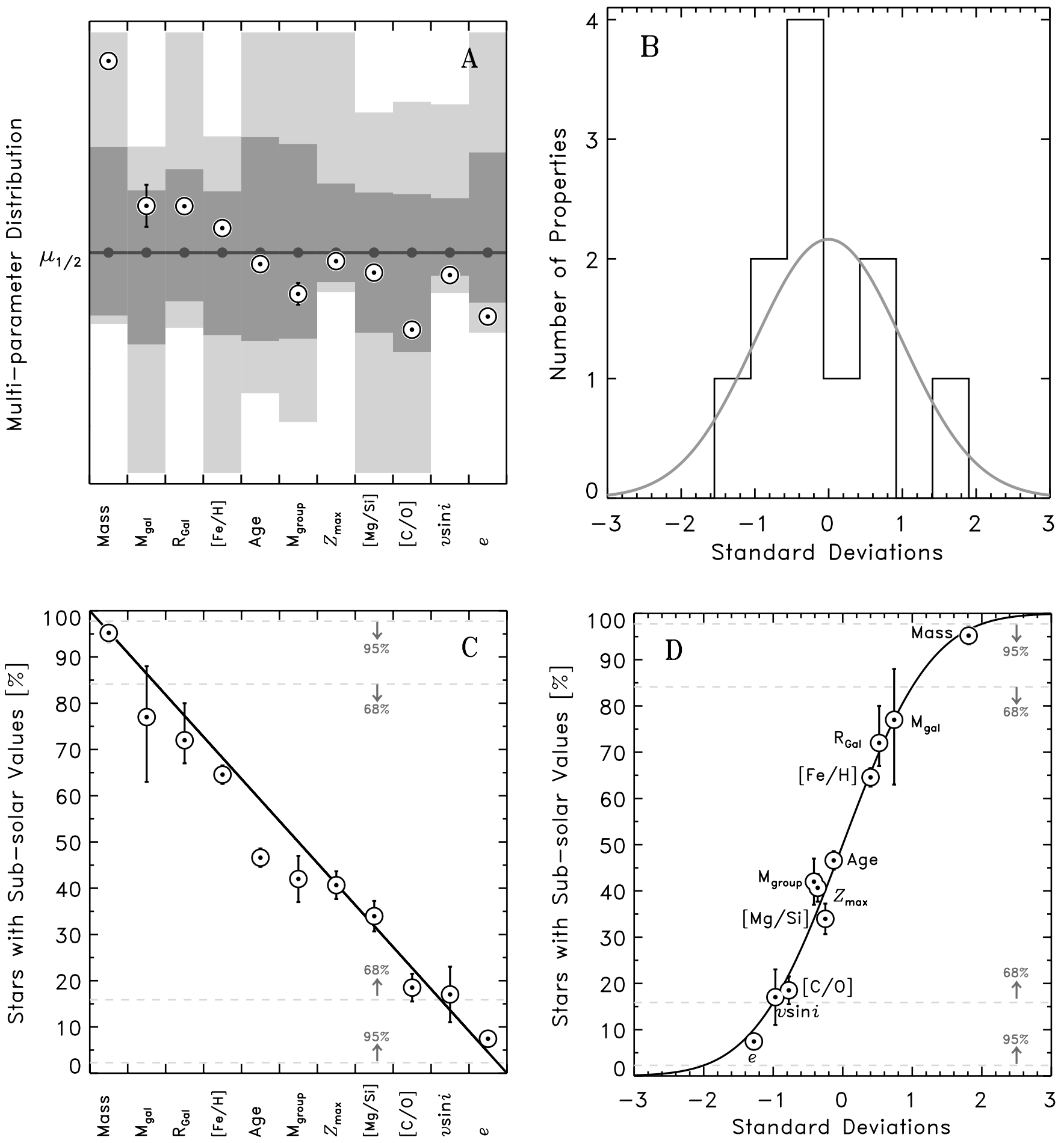

FIG. 13.-Various representations of our main results. (A) Solar values of 11 properties compared to the distribution for each property Each distribution's median value is indicated by a small filled circle. The dark and light gray scale represent the $68 \%$ and $95 \%$ zones, respectively. (B) Histogram of the number of properties as a function of the number of standard deviations the solar value is from the median of that property. The gray curve is a Gaussian probability distribution normalized to 11 parameters. (C) Percentage $n_{i} \%$ of stars with subsolar values as a function of property. The average signal expected from a random star is shown by the solid line (see $\S 4$ ). (D) Percentage $n_{i} \%$ of stars with subsolar values as a function of the number of standard deviations the solar value is from the median of that property. The solid curve is a cumulative Gaussian distribution - if every sample were a Gaussian distribution, every solar dot would sit exactly on the line. Just as in (C), the dashed lines encompass the $68 \%$ and $95 \%$ zones. Similar to the results from Fig. 12, these four panels indicate that the Sun is a typical star. 
TABLE 3

Summary of How the Sun Compares to Other Stars (see Fig. 13)

\begin{tabular}{|c|c|c|c|}
\hline Parameter & Figure & $n_{i} \%$ & Level of Anomaly \\
\hline Mass ........................... & 1 & $95 \% \pm 2 \%$ & of nearby stars are less massive than the Sun \\
\hline Age ............................. & 2 & $53 \% \pm 2 \%$ & of stars in the thin disk of the Galaxy are older than the Sun \\
\hline 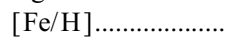 & 3 & $65 \% \pm 2 \%$ & of nearby stars are more iron-poor than the Sun \\
\hline 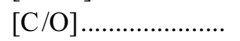 & $4 a$ & $81 \% \pm 3 \%$ & of nearby stars have a higher $\mathrm{C} / \mathrm{O}$ ratio than the Sun \\
\hline$[\mathrm{Mg} / \mathrm{Si}] \ldots \ldots \ldots \ldots \ldots . . . . . .$. & $4 b$ & $66 \% \pm 3 \%$ & of nearby stars have a higher $\mathrm{Mg} / \mathrm{Si}$ ratio than the Sun \\
\hline$v \sin i \ldots \ldots \ldots \ldots \ldots \ldots \ldots \ldots \ldots \ldots \ldots$ & 5 & $83 \% \pm 7 \%$ & of nearby Sun-like-mass stars rotate faster than the Sun \\
\hline e & 6 & $93 \% \pm 1 \%$ & of nearby stars have larger galactic orbital eccentricities than the Sun \\
\hline$Z_{\max } \ldots \ldots \ldots \ldots \ldots \ldots \ldots \ldots$ & 7 & $59 \% \pm 3 \%$ & of nearby stars reach farther from the Galactic plane than the Sun \\
\hline$R_{\mathrm{Gal}} \ldots \ldots \ldots \ldots \ldots \ldots \ldots \ldots \ldots \ldots \ldots \ldots$ & 8 & $72_{-5}^{+8} \%$ & of stars in the Galaxy are closer to the galactic center than the Sun \\
\hline$M_{\mathrm{gal}} \ldots \ldots \ldots \ldots \ldots \ldots \ldots \ldots \ldots \ldots \ldots \ldots \ldots$ & 9 & $77_{-14}^{+11} \%$ & of stars in the universe are in galaxies less massive than the Milky Way \\
\hline 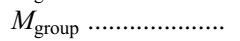 & 10 & $58 \% \pm 5 \%$ & of stars in the universe are in groups more massive than the local group \\
\hline
\end{tabular}

Properties Not Included in the Analysis Because They Are Correlated with the Selected 11 Parameters

\begin{tabular}{|c|c|c|c|}
\hline Mass: $I_{\text {Stellar ...... }}$ & 1 & $94 \% \pm 2 \%$ & of nearby stars are less massive than the Sun \\
\hline Age: $\mathrm{SFR}_{\mathrm{Cosmic}} \ldots \ldots$ & 2 & $86 \% \pm 5 \%$ & of stars in the universe are older than the Sun \\
\hline $\operatorname{Age}^{\mathrm{a}} \ldots \ldots \ldots \ldots \ldots \ldots \ldots$ & $\ldots$ & $55 \% \pm 2 \%$ & of nearby Sun-like-mass stars are older than the Sun \\
\hline$[\mathrm{Fe} / \mathrm{H}]^{\mathrm{b}} \ldots \ldots$. & $\ldots$ & $56 \% \pm 5 \%$ & of nearby stars are more iron-poor than the Sun \\
\hline$v \sin i^{\mathrm{c}} \ldots \ldots \ldots \ldots \ldots \ldots$ & $\ldots$ & $92 \% \pm 5 \%$ & of nearby Sun-like-mass stars rotate faster than the Sun \\
\hline $\log R_{\mathrm{HK}}^{\prime}{ }^{\mathrm{d}} \ldots \ldots \ldots \ldots \ldots$ & $\ldots$ & $51 \% \pm 2 \%$ & of nearby FGKM stars are more chromospherically active \\
\hline$[\mathrm{O} / \mathrm{Fe}] \ldots \ldots \ldots \ldots \ldots \ldots$ & $\ldots$ & $75 \% \pm 3 \%$ & of nearby stars have a lower $\mathrm{O} / \mathrm{Fe}$ ratio than the Sun \\
\hline$R_{\min } \ldots \ldots \ldots \ldots \ldots \ldots \ldots$ & $\ldots$ & $91 \% \pm 1 \%$ & of nearby stars get closer to the Galactic center \\
\hline$v_{\text {LSR }} \ldots \ldots \ldots \ldots \ldots \ldots \ldots \ldots$ & $\ldots$ & $93 \% \pm 1 \%$ & of nearby stars have smaller velocity with respect to the LSR \\
\hline 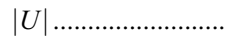 & $\ldots$ & $75 \% \pm 1 \%$ & of nearby stars have larger absolute radial velocity \\
\hline$|V| \ldots \ldots \ldots \ldots \ldots \ldots \ldots \ldots$ & $\ldots$ & $82 \% \pm 1 \%$ & of nearby stars have larger absolute tangential velocity \\
\hline$|W| \ldots \ldots \ldots \ldots \ldots \ldots \ldots \ldots$ & $\ldots$ & $58 \% \pm 1 \%$ & of nearby stars have larger absolute vertical velocity \\
\hline
\end{tabular}

a 1126 stars (A5-K2) from Nordström et al. (2004).

b 91 stars (GK) from Favata et al. (1997).

c 590 stars (F8-K2) from Nordström et al. (2004).

d 866 stars (FGKM) from Wright et al. (2004).

probability that a randomly selected star will be single is $52.8 \% \pm$ $4.5 \%$, which means that about half of stars are single while the other half have one or more companions. Including this in our bootstrap analysis and Monte Carlo simulations (see Appendix $\mathrm{B}, \S \mathrm{B} 1$ ) marginally increases the probability in equation (4) to $33 \% \pm 11 \%$. If the multiplicity data for $246 \mathrm{G}$ dwarfs from Duquennoy \& Mayor (1991) is used instead - the probability that a randomly selected $\mathrm{G}$ dwarf will be single is $37.8 \% \pm 2.9 \%$ then the probability in equation (4) would increase to $34 \% \pm 11 \%$. The inclusion of stellar multiplicity marginally increases our reported probability.

In Figures 6 and 7 of Radick et al. (1998) the Sun's short-term variability as a function of average chromospheric activity appears $\sim 1 \sigma$ low, compared to a distribution of $35 \mathrm{~F} 3-\mathrm{K} 7$ Sunlike stars (Lockwood et al. 1997). Lockwood et al. (2007) suggest that the Sun's small total irradiance variation compared to stars with similar mean chromospheric activity may be due to their limited sample and the lack of solar observations out of the Sun's equatorial plane. We do not include short- or long-term variability (chromospheric or photometric) in Table 3 because of the small size of the Lockwood et al. (2007) sample. We also do not include the chromospheric index $R_{\mathrm{HK}}^{\prime}$ (see Table 3, bottom panel) as one of our 11 properties because of its correlation with the chromospheric ages of our sample.

\section{DISCUSSION AND INTERPRETATION}

The probability $P_{\mathrm{MC}}\left(\leq \chi_{\odot}^{2}\right)=0.29 \pm 0.11$ classifies the Sun as a typical star. How robust is this result? The probability of finding a star with a $\chi^{2}$ lower than or equal to $\chi_{\odot}^{2}$ depends on the properties selected for the analysis (see problem 3 in $\S 1$ ). For example, if we had chosen to consider only mass and eccentricity data, this analysis would yield $P_{\mathrm{MC}}\left(\chi^{2} \leq \chi_{\odot}^{2}\right)=0.94 \pm 0.4$; i.e., the Sun would appear mildly $(\sim 2 \sigma)$ anomalous. If on the other hand, we had chosen to remove mass and eccentricity from the analysis, we would obtain $P_{\mathrm{MC}}\left(\chi^{2} \leq \chi_{\odot}^{2}\right)=0.07 \pm 0.04$, which is anomalously low. The most common cause of such a result is the overestimation of error bars. The next most common cause is the preselection of properties known to have $n_{i} \% \sim 50 \%$.

Gustafsson (1998) discussed the atypically large solar mass and proposed an anthropic explanation - the Sun's high mass is probably related to our own existence. He suggested that the solar mass could hardly have been greater than $\sim 1.3 M_{\odot}$ since the main-sequence lifetime of a $1.3 M_{\odot}$ star is $\sim 5$ billion years (Clayton 1983). He also discussed how the dependence of the width of the circumstellar habitable zone on the host star's mass probably favors host stars within the mass range $0.8-1.3 M_{\odot}$.

Our property selection criteria is to have the largest number of maximally independent properties that have a plausible correlation with habitability and ones for which a representative stellar sample could be assembled. Our joint analysis does not weight any parameter more heavily than any other. If the only properties associated with habitability are mass and eccentricity, then we have diluted a $\sim 2 \sigma$ signal that would be consistent with Gustafsson's proposed anthropic explanation.

Our analysis points in another direction. If mass and eccentricity were the only properties associated with habitability, then the solar values for the remaining nine properties would be consistent with noise. However, a joint analysis of just the remaining nine properties produces a $\chi_{\odot .9}^{2}=3.6 \pm 0.4$ and the anomalously low probability $P\left(\leq \chi_{\odot, 9}^{2}\right)=0.07 \pm 0.04$, which suggests that the nine properties are unlikely to be the properties of a star selected at random with respect to these properties. 
The $\chi^{2}$ fit of the 11 points in Figure $13 c$ to the diagonal line yields a fit that is substantially better then the fit of the remaining nine properties to equation (B1) with $N=9$. In other words, the joint analyis suggests that although mass and eccentricity are the most anomalous solar properties, it is unlikely that they are associated with habitability, because without them it is unlikely that the remaining solar properties are just noise. Thus, the Sun, despite its mildly $(\sim 2 \sigma)$ anomalous mass and eccentricity, can be considered a typical, randomly selected star.

There may be stellar properties crucial for life that were not tested here. If we have left out the most important properties, with respect to which the Sun is atypical, then our Sun-is-typical conclusion will not be valid. If we have sampled all properties associated with habitability, our Sun-is-typical result suggests that there are no special requirements on a star for it to be able to host a planet with life.

\section{CONCLUSIONS}

We have compared the Sun to representative stellar samples for 11 properties. Our main results are as follows:

1. Stellar mass and Galactic orbital eccentricity are the most anomalous properties. The Sun is more massive than $95 \% \pm 2 \%$ of nearby stars and has a Galactic orbital eccentricity lower than $93 \% \pm 1 \%$ FGK stars within 40 pc.

2. Our joint bootstrap analysis yields a solar $\chi^{2}$ of $\chi_{\odot}^{2}=$ $8.39 \pm 0.96$ and a solar reduced $\chi^{2}$ of $\chi_{\odot}^{2} / 11=0.76 \pm 0.09$. The probability offinding a star with a $\chi^{2}$ lower than or equal to solar $P_{\mathrm{MC}}\left(\leq \chi_{\odot}^{2}=8.39 \pm 0.96\right)=0.29 \pm 0.11$.

To our knowledge, this is the most comprehensive and quantitative comparison of the Sun with other stars. We find that taking all 11 properties together, the Sun is a typical star. This finding is largely in agreement with Gustafsson (1998); however, our results undermine the proposition that an anthropic explanation is needed for the comparatively large mass of the Sun.

Further work could encompass the inclusion of other properties potentially associated with habitability. Another improvement would come when larger stellar samples become available for which all properties could be derived, instead of using different samples for different properties as was done here. In addition, research in the molecular evolution that led to the origin of life may, in the future, be able to provide more clues as to which stellar properties might be associated with our existence on Earth, orbiting the Sun.

We would like to thank Charles Jenkins for clarifying discussions of statistics, particularly on how to include stellar multiplicity, and Martin Asplund and Jorge Meléndez for discussions of elemental abundances. J. A. R. acknowledges an RSAA Ph.D. research scholarship. M. P. acknowledges the financial support of the Australian Research Council. E. G. acknowledges the financial support of the Finnish Cultural Foundation.

\section{APPENDIX A}

\section{PROPERTY CORRELATIONS}

The $\chi^{2}$ formalism and the use of the $\chi^{2}$ distribution to obtain $P\left(<\chi_{\odot}^{2} \mid N\right)$-improved using Monte Carlo simulations in $\S 3.2$ to obtain $P_{\mathrm{MC}}\left(\leq \chi_{\odot}^{2}\right)$ - assumes that each parameter is independent of the others. In selecting our 11 properties we have selected properties that are maximally independent based on plotting property 1 versus property 2 for the same stars. We show seven such plots in this appendix.

If there are correlations between the analyzed properties, then the number of degrees of freedom $N$ could drop from 11 to $\sim 10.5$ (see Fig. 12). Some properties have been excluded from the analysis due to a correlation with another property in the analysis.

\section{A1. ELEMENTAL RATIOS}

In Figure 14 we show the distribution for carbon to oxygen ratio [C/O] versus the magnesium to silicon ratio [ $\mathrm{Mg} / \mathrm{Si}]$ of $176 \mathrm{FG}$ stars.

\section{A2. MASS, AGE, AND ROTATIONAL VELOCITY}

In Figure 15 we show four correlation plots for mass, chromospheric age, rotational velocity, and $v$ sin $i$. We use the stars common to both Wright et al. (2004) and Valenti \& Fischer (2005) for which these observables are available.

\section{A3. GALACTIC ORBITAL PARAMETERS}

In Figure 16 the Galactic orbital eccentricity $(e)$ and the magnitude of the galactic orbital velocities with respect to the local standard of rest $\left(v_{\text {LSR }}\right)$ are strongly correlated (see Fig. 6). We selected $e$ instead of $v_{\text {LSR }}$ because of its near independence of the maximum height above the galactic plane $\left(Z_{\max }\right)$.

\section{APPENDIX B}

\section{IMPROVED ESTIMATES OF $\chi_{\odot}^{2}$ AND $P\left(<\chi_{\odot}^{2}\right)$}

In $\S 3.2$, with 11 degrees of freedom, the reduced $\chi^{2}$ from equation (4) is $\chi_{\odot}^{2} / 11=0.72_{-0.03}^{+0.01}$. Since $\chi_{\odot}^{2} / 11<1$, the Sun's properties are consistent with the Sun being a randomly selected star.

To improve on this preliminary analysis (but with a similar conclusion), as mentioned in $\S 3.2$, we employ a bootstrap analysis (Efron 1979 ) to randomly resample data (with replacement) and derive a more accurate estimate of $\chi_{\odot}^{2}$. Because the bootstrap is a nonparametric method, the distributions need not be Gaussian. 


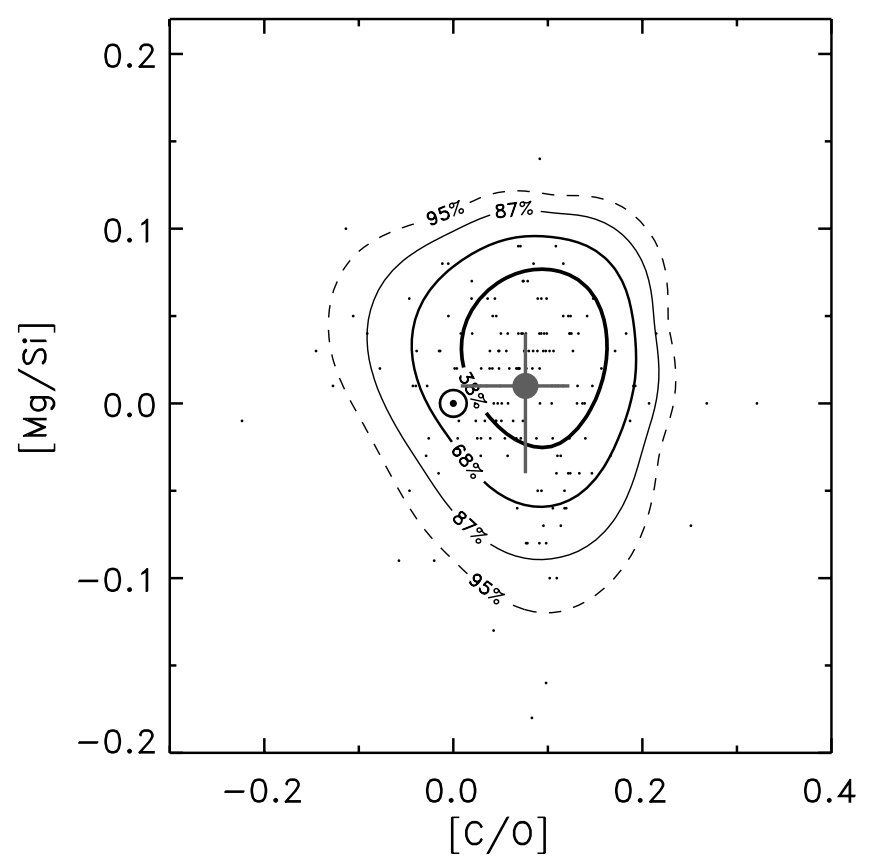

Fig. 14. - Carbon to oxygen ratio [C/O] vs. magnesium to silicon ratio [ $\mathrm{Mg} / \mathrm{Si}]$ of $176 \mathrm{FG}$ stars with abundances for these elements (Reddy et al. 2003). In Figs. 4c and $4 d$ we showed that the $[\mathrm{C} / \mathrm{O}]$ and $[\mathrm{Mg} / \mathrm{Si}]$ distributions are largely independent of $[\mathrm{Fe} / \mathrm{H}]$. Here we show that these distributions are also largely independent of each other. Note that in this comparison we only use the data from Reddy et al. (2003) since it is the largest available sample with C, O, Mg, and Si abundances.

For every iteration, each parameter's stellar distribution is randomly resampled and a $\chi_{\odot}^{2}$ value is calculated using equation (1). The uncertainties $\sigma_{\odot, i}$ of the solar values $x_{\odot, i}$ are also included in the bootstrap method; for every iteration, the solar value for each parameter is replaced in equation (1) by a randomly selected value from a normal distribution with median $\mu_{1 / 2, i}=x_{\odot, i}$ and standard deviation $\sigma_{\odot, i}$. The process was iterated 100,000 times, although the resulting distribution varies very little once the number of iterations reaches $\sim 10,000$.

The median of this distribution and the error on the median yields our improved value for the reduced $\chi^{2}$ (Fig. 11). The uncertainty of the median of each resampled distribution varies inversely proportionally to the square root of the number of stars in the distribution, $\Delta \mu_{1 / 2, i} \propto 1 /\left(N_{\star, i}\right)^{1 / 2}$. In other words, median values are less certain for smaller samples, and this uncertainty is included in our improved estimate of $\chi_{\odot}^{2}$ and its uncertainty.

We find the probability of finding a star with a $\chi_{\star}^{2}$ value lower than the solar $\chi_{\odot}^{2}$ for $N=11$ degrees of freedom in the standard way (Press et al. 1992) and obtain

$$
P\left(<\chi_{\odot}^{2}=7.88_{-0.30}^{+0.08} \mid 11\right)=0.28_{-0.03}^{+0.01} .
$$

To improve our estimate of the probability of finding a star with lower $\chi^{2}$ value than the Sun, we perform a Monte Carlo simulation (Metropolis \& Ulam 1949) to calculate an estimate of each star's $\chi^{2}$ value $\left(\chi_{\star}^{2}\right)$. For every iteration, we randomly select a star from each stellar distribution. We then calculate its $\chi_{\star}^{2}$ value by replacing the solar value $x_{\odot, i}$ with that star's value $x_{\star, i}$ in equation (1). This process was repeated 100,000 times to create our Monte Carlo stellar $\chi^{2}$ distribution. Stars were randomly selected with replacement; thus, the simulated $\chi^{2}$ distribution accounts for small number statistics and non-Gaussian distributions. The probability of finding a star with $\chi^{2}$ lower than or equal to solar is $P_{\mathrm{MC}}=0.29 \pm 0.11$.

The results of our analysis for the solar $\chi_{\odot}^{2}$ values and the probabilities $P\left(<\chi_{\odot}^{2}\right)$ are summarized in Table 2 .

\section{B1. ADDITION OF A DISCRETE PARAMETER}

In $\S 4$ we discuss the addition of stellar multiplicity to our analysis. Since stellar multiplicity cannot easily be approximated by a onesided Gaussian ( particularly because the Sun is on the edge of the distribution, i.e., it is of multiplicity one), we modified our Monte Carlo procedure to include this discrete parameter. The likelihood of observing a particular $\chi^{2}$ for the 11 parameters is

$$
\exp \left(-\frac{1}{2} \sum_{i=1}^{11} \chi_{i}^{2}\right) \text {. }
$$

We take the probability $p(1)$ of a star being a single star to be $53.8 \% \pm 4.5 \%$, obtained from our sample of nearby stars $(\S 2.1)$. The likelihood $L$ of observing a particular $\chi^{2}$ and $p(1)$ is the product

$$
L=p(1) \exp \left(-\frac{1}{2} \sum_{i=1}^{11} \chi_{i}^{2}\right) .
$$



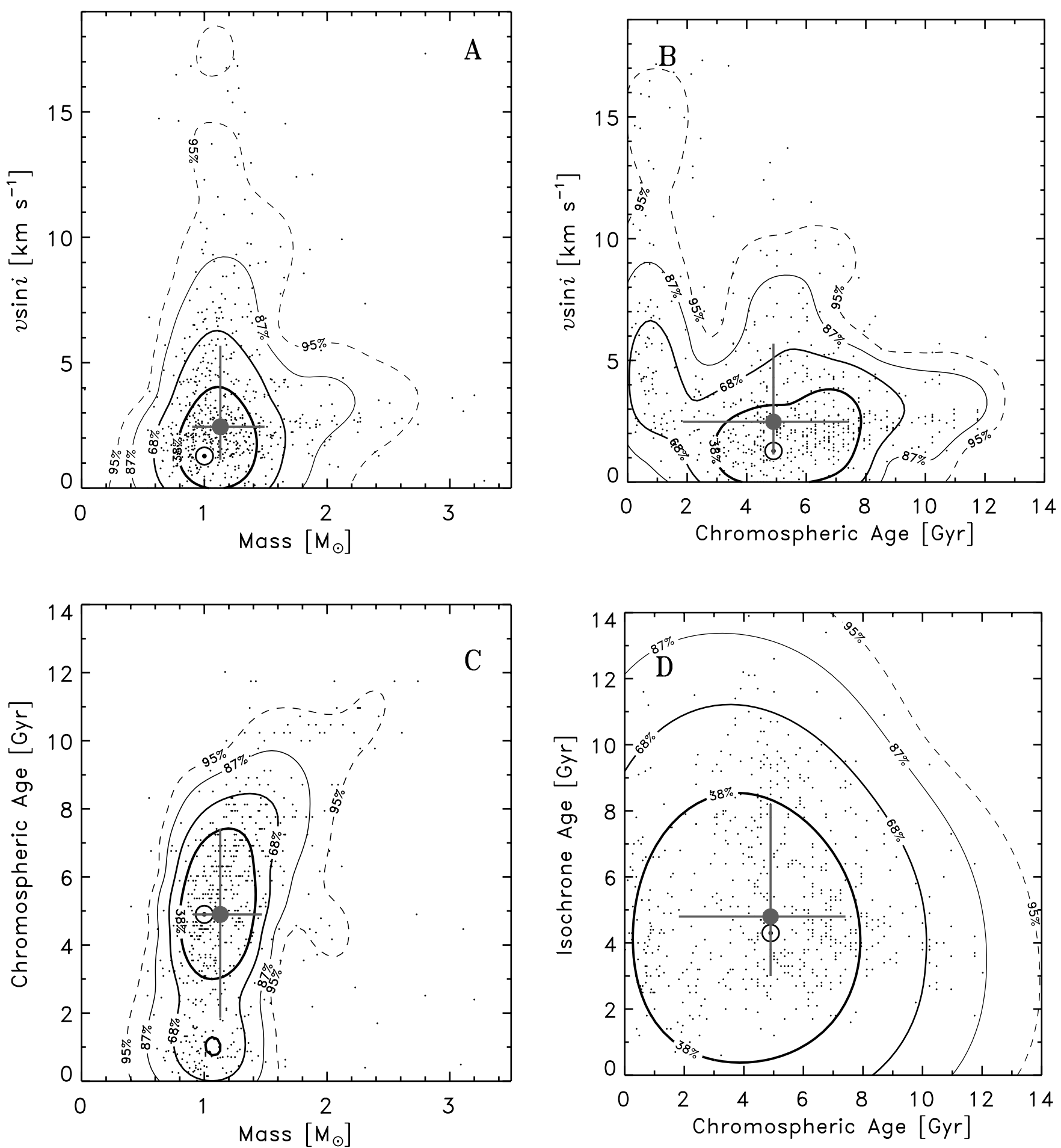

FIG. 15.-Correlation plots between various properties. For all four panels we use the stars common to both Wright et al. (2004) and Valenti \& Fischer (2005). (A): Mass vs. rotational velocity $v \sin i$ for 713 FGK stars. This panel shows the degree of correlation between mass and $v \sin i$. See Gray (2005) for a stronger correlation between these two variables when a larger mass range and more active stars are kept in the sample. To minimize the effect of this correlation on our analysis, we restrict the range of mass in Fig. 5 to $0.9-1.1 M_{\odot}$. (B): Chromospheric age vs. $v \sin i$ for 641 FGK stars. The lack of correlation between chromospheric determined ages and rotational velocities is shown. (C) No strong correlation between mass and chromospheric age for 639 FGK stars. (D) Ages of 637 stars determined by the chromospheric method vs. their ages from the isochrone method.

Taking logarithms we can then compute the distribution of the statistic $S$, where

$$
S=\ln p(1)-\frac{1}{2} \sum_{i=1}^{11} \chi_{i}^{2}
$$

The distribution of $S$ allows us to obtain the results for the multiplicity reported at the end of $\S 4$. 

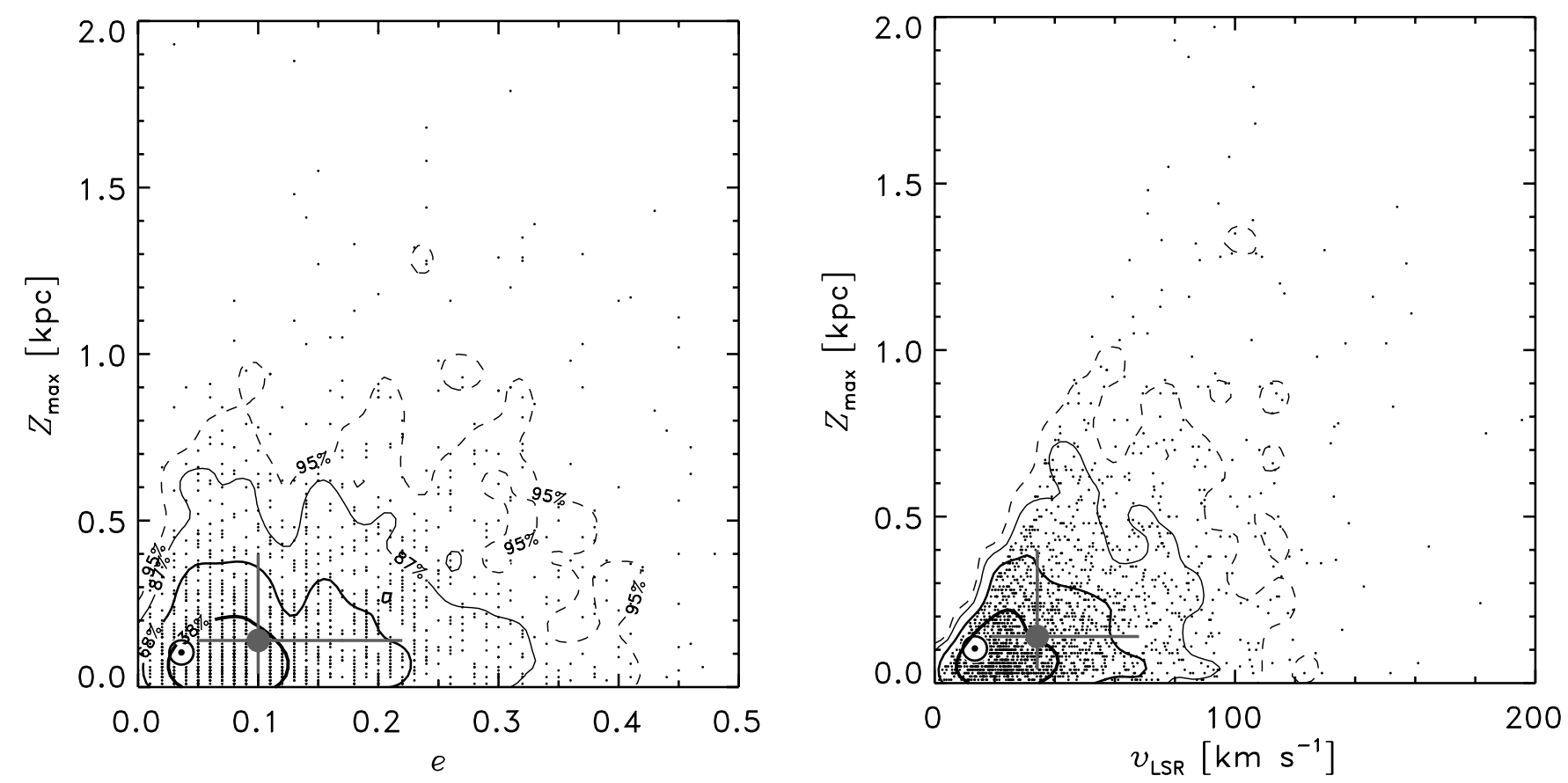

FIG. 16. - Left: Galactic orbital eccentricity $e$ vs. $Z_{\max }$ for 1987 FGK stars within $40 \mathrm{pc}$ (Nordström et al. 2004). The orbital eccentricity is not correlated with $Z_{\max }$. Right: $v_{\text {LSR }}$ vs. $Z_{\max }$ for the same stars. Because $v_{\text {LSR }}$ is more strongly correlated with $Z_{\max }$ than eccentricity, eccentricity has been selected for the joint analysis instead of $v_{\text {LSR }}$. As in Fig. 4, the contours correspond to $38 \%, 68 \%, 82 \%$, and $95 \%$.

Allègre, C. J., Manhès, G., \& Göpel, C. 1995, Geochim. Cosmochim. Acta, 59, 1445 Allende Prieto, C. 2006, preprint (astro-ph/0612200)

Allende Prieto, C., Lambert, D. L., \& Asplund, M. 2001, ApJ, 556, L63

Asplund, M., Grevesse, N., \& Sauval, A. J. 2005, in ASP Conf. Ser. 336, Cosmic Abundances as Records of Stellar Evolution and Nucleosynthesis, ed. T. G. Barnes III \& F. N. Bash (San Francisco: ASP), 25

Bahcall, J. N., \& Soneira, R. M. 1980, ApJS, 44, 73

Bell, E. F., \& de Jong, R. S. 2001, ApJ, 550, 212

Bensby, T., \& Feltzing, S. 2006, MNRAS, 367, 1181

Bensby, T., Feltzing, S., Lundström, I., \& Ilyin, I. 2005, A\&A, 433, 185

Bertelli, G., \& Nasi, E. 2001, AJ, 121, 1013

Carter, B. 1983, Philos. Trans. R. Soc. London A, 310, 347

Clayton, D. D. 1983, Principles of Stellar Evolution and Nucleosynthesis (Chicago: Univ. Chicago Press)

Courteau, S., \& van den Bergh, S. 1999, AJ, 118, 337

Dehnen, W., \& Binney, J. J. 1998, MNRAS, 298, 387

Driver, S. P., Phillipps, S., Davies, J. I., Morgan, I., \& Disney, M. J. 1994, MNRAS, 268, 393

Duquennoy, A., \& Mayor, M. 1991, A\&A, 248, 485

Edvardsson, B., Andersen, J., Gustafsson, B., Lambert, D. L., Nissen, P. E., \& Tomkin, J. 1993a, A\&A, 275, 101 1993b, A\&AS, 102, 603

Efron, B. 1979, Ann. Stat., 7, 1

Eisenhauer, F., et al. 2005, ApJ, 628, 246

Eke, V. R., et al. 2004, MNRAS, 355, 769

Favata, F., Micela, G., \& Sciortino, S. 1996, A\&A, 311, 951 1997, A\&A, 323, 809

Feltzing, S., Holmberg, J., \& Hurley, J. R. 2001, A\&A, 377, 911

Flynn, C., Holmberg, J., Portinari, L., Fuchs, B., \& Jahreiß, H. 2006, MNRAS, 372,1149

Flynn, C., Sommer-Larsen, J., \& Christensen, P. R. 1996, MNRAS, 281, 1027

Fuhrmann, K. 2008, MNRAS, 384, 173

Gonzalez, G. 1999a, MNRAS, 308, 447 1999b, Astron. Geophys., 40, 25

Gonzalez, G., Brownlee, D., \& Ward, P. 2001, Icarus, 152, 185

Gould, A., Bahcall, J. N., \& Flynn, C. 1996, ApJ, 465, 759

Gray, D. F. 2005, The Observation and Analysis of Stellar Photospheres (Cambridge: Cambridge Univ. Press)

Grether, D., \& Lineweaver, C. H. 2006, ApJ, 640, 1051 2007, ApJ, 669, 1220

Gustafsson, B. 1998, Space Sci. Rev., 85, 419

Gustafsson, B., Karlsson, T., Olsson, E., Edvardsson, B., \& Ryde, N. 1999, A\&A, 342, 426

\section{EFERENCES}

Hernandez, X., Valls-Gabaud, D., \& Gilmore, G. 2000, MNRAS, 316, 605 Hogg, D. W., Blanton, M. R., Roweis, S. T., \& Johnston, K. V. 2005, ApJ, 629, 268 Hopkins, A. M., \& Beacom, J. F. 2006, ApJ, 651, 142

Jarrett, T. H., Chester, T., Cutri, R., Schneider, S. E., \& Huchra, J. P. 2003, AJ, 125,525

Kasting, J. F., Whitmire, D. P., \& Reynolds, R. T. 1993, Icarus, 101, 108 Kroupa, P. 2002, Science, 295, 82

Kroupa, P., \& Weidner, C. 2005, in The Initial Mass Function 50 Years Later, ed. E. Corbelli, F. Palla, \& H. Zinnecker (ASSL Vol. 327; Dordrecht: Springer), 175

Kuchner, M. J., \& Seager, S. 2005, preprint (astro-ph/0504214)

Lineweaver, C. H., Fenner, Y., \& Gibson, B. K. 2004, Science, 303, 59

Lockwood, G. W., Skiff, B. A., Henry, G. W., Henry, S., Radick, R. R., Baliunas, S. L., Donahue, R. A., \& Soon, W. 2007, ApJS, 171, 260

Lockwood, G. W., Skiff, B. A., \& Radick, R. R. 1997, ApJ, 485, 789

Loveday, J. 2000, MNRAS, 312, 557

Metropolis, N., \& Ulam, S. 1949, J. Am. Stat. Assoc., 44, 335

Metzger, M. R., Caldwell, J. A. R., \& Schechter, P. L. 1998, AJ, 115, 635

Meyer, S. L. 1975, Data Analysis for Scientists and Engineers (New York: Wiley)

Nordström, B., et al. 2004, A\&A, 418, 989

Press, W. H., Teukolsky, S. A., Vetterling, W. T., \& Flannery, B. P. 1992, Numerical Recipes in FORTRAN: The Art of Scientific Computing (2nd ed.; Cambridge: Cambridge Univ. Press)

Radick, R. R., Lockwood, G. W., Skiff, B. A., \& Baliunas, S. L. 1998, ApJS, 118,239

Ramírez, I., Allende Prieto, C., \& Lambert, D. L. 2007, A\&A, 465, 271

Reddy, B. E., Tomkin, J., Lambert, D. L., \& Allende Prieto, C. 2003, MNRAS, 340,304

Reid, I. N. 2002, PASP, 114, 306

Reid, I. N., Turner, E. L., Turnbull, M. C., Mountain, M., \& Valenti, J. A. 2007, ApJ, 665, 767

Rocha-Pinto, H. J., Maciel, W. J., Scalo, J., \& Flynn, C. 2000a, A\&A, 358, 850 Rocha-Pinto, H. J., Scalo, J., Maciel, W. J., \& Flynn, C. 2000b, ApJ, 531, L115 Schechter, P. 1976, ApJ, 203, 297

Silk, J. 2007, Astron. Geophys., 48, 30

Soderblom, D. R. 1983, ApJS, 53, 1 . 1985, AJ, 90, 2103

Truran, J. W., Jr., \& Heger, A. 2005, Origin of the Elements, ed. A. M. Davis (Amsterdam: Elsevier), 1

Valenti, J. A., \& Fischer, D. A. 2005, ApJS, 159, 141

van den Bergh, S. 2000, The Galaxies of the Local Group (Cambridge: Cambridge Univ. Press)

Wright, J. T., Marcy, G. W., Butler, R. P., \& Vogt, S. S. 2004, ApJS, 152, 261 